\title{
TV/Series
}

$17 \mid 2020$

Séries : les sens de l'Histoire

\section{Cuéntame cómo pasó et Conta-me como foi : les dictatures ibériques à travers le regard des petits «Carlitos »}

\section{Cécile Gonçalves}

\section{OpenEdition}

Journals

Édition électronique

URL : http://journals.openedition.org/tvseries/4092

DOI : $10.4000 /$ tvseries.4092

ISSN : 2266-0909

Éditeur

GRIC - Groupe de recherche Identités et Cultures

Référence électronique

Cécile Gonçalves, «Cuéntame cómo pasó et Conta-me como foi : les dictatures ibériques à travers le regard des petits «Carlitos » », TV/Series [En ligne], 17 | 2020, mis en ligne le 24 juin 2020, consulté le 06 septembre 2020. URL : http://journals.openedition.org/tvseries/4092 ; DOI : https://doi.org/ 10.4000/tvseries.4092

Ce document a été généré automatiquement le 6 septembre 2020

\section{(†) $\Theta$}

TV/Series est mis à disposition selon les termes de la licence Creative Commons Attribution - Pas d'Utilisation Commerciale - Pas de Modification 4.0 International 


\title{
Cuéntame cómo pasó et Conta-me como foi : les dictatures ibériques à travers le regard des petits «Carlitos»
}

\author{
Cécile Gonçalves
}

La série TV espagnole Cuéntame cómo pasó ${ }^{1}$, qu'on peut traduire par «Raconte-moi comment ça s'est passé » a été l'un des plus gros succès populaires enregistrés par la Radio y Televisión Española (RTVE) ces quarante dernières années. Diffusée depuis le 13 septembre 2001, tous les jeudis soirs en prime time, elle a vu ses chiffres d'audience croître de semaine en semaine, chaque épisode attirant en moyenne 4,7 millions de téléspectateurs fidèles ${ }^{2}$. Selon les données publiées par El Estudio General de Medios (EGM), Cuéntame a ainsi su séduire entre $12,4 \%$ et $40 \%$ des femmes responsables des achats de moins de 50 ans (FRDA-50) ${ }^{3}$. Le succès a été tel en Espagne que le concept de la série phare de la RTVE a été exporté dans plusieurs pays à travers le monde : au Portugal (Conta-me como foi), en Italie (Raccontami), en République Tchèque, en Turquie, en Grèce, au Mexique, en Argentine (Cuéntame cómo pasó), au Chili (Los 80), en Équateur (Parece que fue ayer) ou aux États-Unis (Remember When). Toutefois, pour notre propos, faute d'avoir vu toutes ces adaptations, notre comparaison se limitera uniquement aux versions ibériques. 
Fig. 1. Cuéntame, année 1968

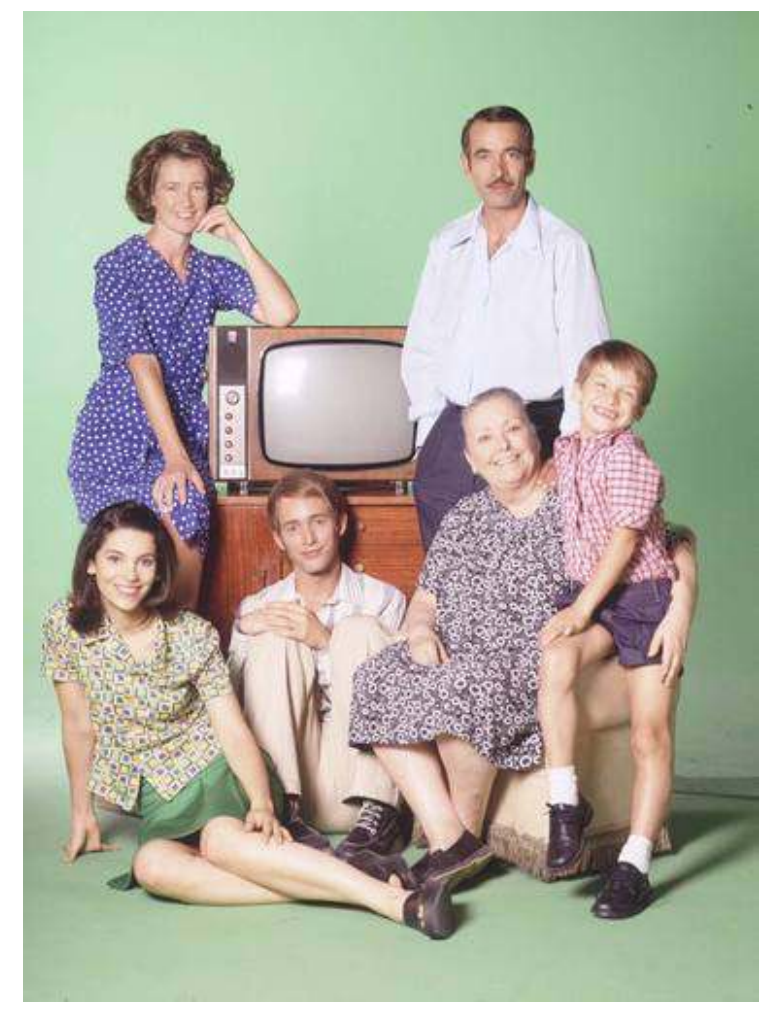

2 La série originale a été créée à l'occasion des vingt-cinq ans de la transition démocratique espagnole par Miguel Angel Bernardeau, de la société Ganga Producciones, et compte pas moins de 368 épisodes courant sur 20 saisons ${ }^{4}$. Cuéntame cómo pasó est, pour reprendre l'expression de Martin Winckler ${ }^{5}$, une "téléfiction » c'est-à-dire qu'il s'agit d'une fiction en épisodes pensée et conçue spécifiquement dans les conditions de production et de diffusion de la télévision. Elle emprunte beaucoup au genre du soap opera ou, comme disent nos cousins Québécois, au "roman-savonnette», genre particulièrement prisé du grand public, même si elle s'avère au final mieux écrite et mieux produite. Étant tournée avec des moyens propres au cinéma, elle se cantonne à une périodicité hebdomadaire. Cuéntame est, en effet, une série hebdomadaire de 70 minutes avec des personnages récurrents et des épisodes autonomes : à savoir, des histoires complètes, en un seul épisode ayant une atmosphère particulière, une thématique propre. Mais, Cuéntame est également un feuilleton à la manière d'un soap. Les soap sont, quant à eux, des mélodrames quotidiens, des feuilletons sentimentaux, lancés dans les années 1950 par des fabricants de lessive pour promouvoir leurs marques sur le petit écran (d'où leur nom, "soap » désignant le savon). Ce genre, écrit Jean Bianchi, «c'est la télévision au long cours : le feuilleton-type est bâti pour durer des années, cinquante deux-semaines par an, à raison d'un épisode quotidien d'une heure du lundi au vendredi $»^{6}$. Les « romans savonnette» sont les uniques narrations dans lesquelles les personnages vieillissent en même temps que le public. La singularité de ce format participe aux interactions entre le public et la fiction elle-même. À ce propos, rajoute Marine Legagneur ${ }^{7}$, le soap

«se caractérise surtout par la proximité entretenue avec les spectateurs, par le biais de la mise en scène du quotidien social de héros familiers, qui trouve un écho dans celui du public, et par la prédictibilité et le phénomène d'identification que cette proximité engendre ». 


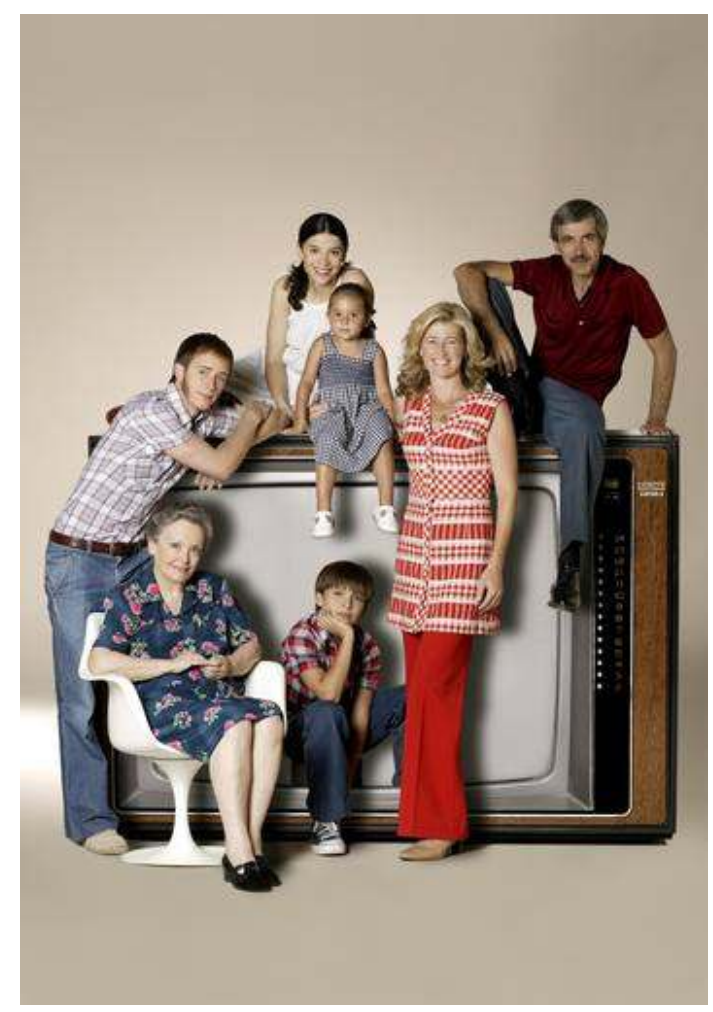

Cuéntame propose aux téléspectateurs, comme pourrait le faire un soap opera, une immersion dans la vie quotidienne des Espagnols sous le franquisme tardif. Démarrant son récit en 1968, elle s'attarde, en combinant micro et macrohistoire, sur les évènements marquants de l'histoire contemporaine du pays comme la Révolution des ๔illets survenue le 25 avril 1974 au Portugal, la mort du Général Francisco Franco dit el Caudillo le 20 novembre 1975, la mise en route de la transition démocratique, la Movida des années 1980, en passant par les attentats de l'ETA ou la tentative de coup d'État du 23 février 1981.

Conta-me como foi ${ }^{8}$ est donc la version portugaise de Cuéntame cómo pasó. Espérant atteindre les records d'audience enregistrés par son homologue, Luis Andrade, directeur des programmes de la Radio-télévision du Portugal (RTP) en 2003, a souhaité l'adapter pour le public portugais dans la mesure où l'histoire contemporaine de ces deux peuples se partageant la péninsule ibérique est très proche, sans pour autant être identique. En effet, si Conta-me como foi renferme de nombreuses similitudes tant du point de vue des arcs narratifs scénaristiques que de la diégèse, la Guerre coloniale (1961-1974) constitue néanmoins la toile de fond qui sous-tend l'action marquant immanquablement une prise de distance par rapport à l'originale espagnole. Ceci étant dit, l'adaptation au contexte local a été possible dans la mesure où le pays de Luis Camões a également subi une dictature national-catholique entre 1932 et 1974. L'« État Nouveau » instauré par António de Oliveira Salazar en 1933 n'a été renversé que le 25 avril 1974 grâce au coup d'État militaire du Mouvement des Forces Armées, resté dans la mémoire collective comme le Mouvement des «Capitaines d'Avril » qui souhaitait en finir avec l'envoi de contingents dans l'Outremer. La population lisboète ayant rejoint 
les militaires pour soutenir leur action, le putsch est devenu « Révolution des Eillets » et a ouvert la voie à la transition démocratique.

5 La série, réalisée par Fernando Avila, fut diffusée sur la première chaîne du service public portugais (RTP 1) entre 2007 et 2009. Comptant 104 épisodes de 50 minutes chacun répartis sur cinq saisons, elle n'a toutefois pas connu auprès du public le succès de Cuéntame $e^{9}$ malgré un casting prestigieux (Miguel Guilherme, Rita Blanco, Luís Ganito, Catarina Avelar) et des critiques élogieuses dans l'ensemble de la presse ${ }^{10}$. Preuve de ce semi-échec, Conta-me a été reprogrammée en deuxième partie de soirée, horaire moins noble, dès la deuxième saison.

Fig. 3. Conta-me

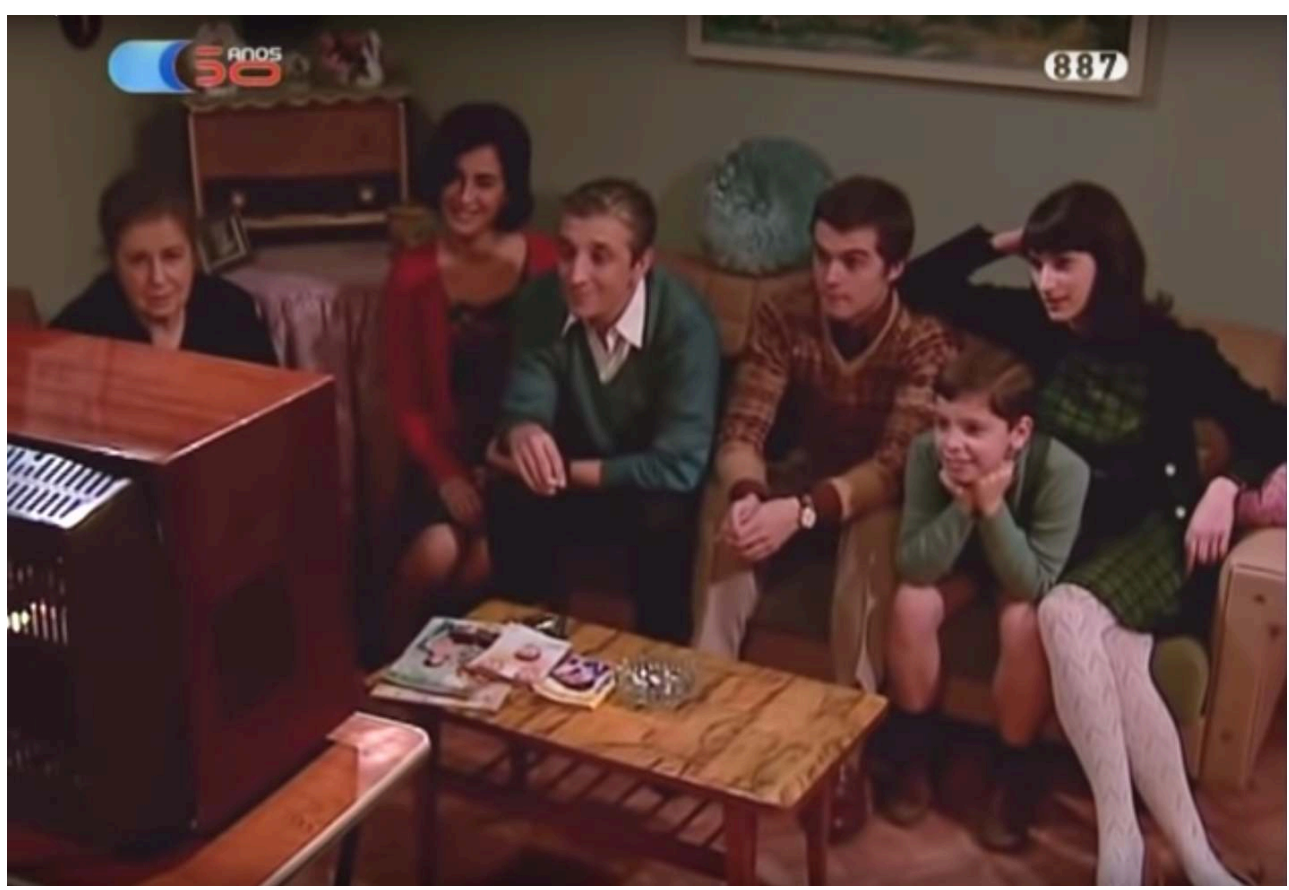

Contrairement à la version espagnole, le récit de Conta-me s'arrête le 25 avril 1974, jour de la Révolution des 〔モillets ${ }^{11}$. Toutefois, dans les deux versions, la trame narrative est la même : il s'est agi de donner à voir le passage d'un régime dictatorial à la démocratie représentative. Les deux séries partagent la volonté de ressusciter par le biais de la fiction des modes de vie disparus avec l'impulsion du processus démocratique. Le schéma narratif suivi est quasiment identique, tout en ayant été soigneusement adapté aux contextes politique et social locaux. Cuéntame et Conta-me sont des analepses ou " flashbacks » qui ont construit leurs scenarii comme un «voyage dans le temps » et un récit-estampes autour de familles particulières. Ces réalisations se caractérisent par " un statisme narratif poussé à l'extrême ${ }^{12}$ " et une visée ouvertement " costumbrista ${ }^{13}$ ", les aventures des petits "Carlitos » n'étant qu'un prétexte pour dépeindre la vie et les mœurs des habitants des quartiers populaires où se situe l'action. Autrement dit, à travers les destinées particulières des familles Alcántara et Lopes, Cuéntame et Conta-me souhaitent incarner le processus de changement politico-social qui a affecté l'ensemble de la péninsule ibérique à la fin des années 1960. Les auteurs ont voulu restituer le pittoresque de l'époque, sa couleur locale. Dans cette étude, on cherchera, par conséquent, à savoir si leur travail a été motivé par une quelconque forme de "saudade» - nostalgie - envers les franquisme et salazarisme tardifs tant les souvenirs 
rapportés par les enfants qui servent de guide à la narration, les deux «Carlitos », qui exaltent le bonheur et la joie de vivre. Il s'est probablement agi pour les différents auteurs de sauver de l'oubli des modes d'être-au-monde disparus qui semblent être considérés par les showrunners comme préférables à ceux du temps présent ${ }^{14}$.

Fig. 4. Conta-me

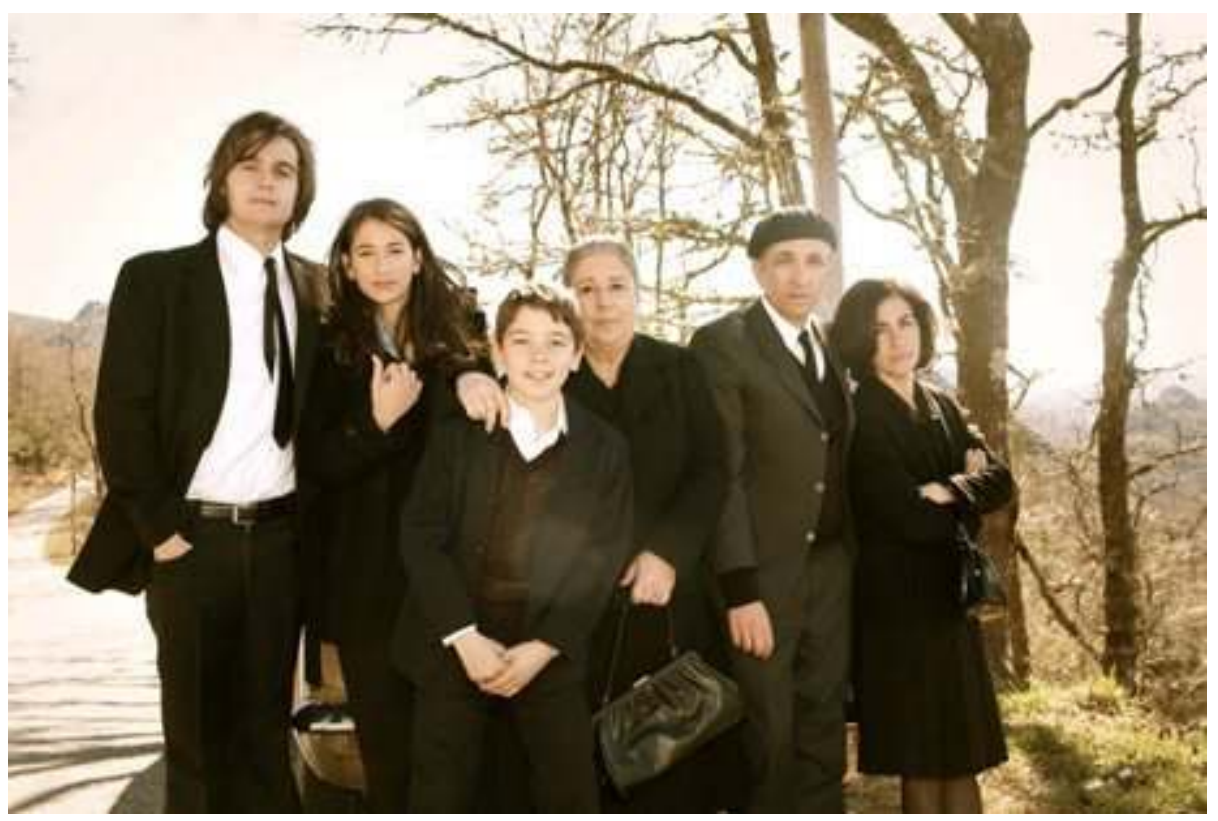

En situant leur action respective dans le cadre d'un quartier périphérique fictif de Madrid baptisé San Genaro et dans un «bairro social " (" quartier populaire ») tout aussi fictif de Lisbonne, les deux séries ont nourri l'ambition de décrire le quotidien d'une famille issue de la classe moyenne sous les franquisme et salazarisme tardifs. Elles dépeignent ainsi deux véritables success stories entrepreneuriales. Venus du monde rural, les deux couples élèvent tant bien que mal leurs enfants nés dans la capitale. Pour les aider à gérer les affres de la vie quotidienne la mère de Mercedes/Margarida, Herminia, s'est installée dans l'appartement familial. La structure familiale et les professions du couple de la version espagnole ont également été reproduites dans la version lusitanienne: le père, Antonio/António, cumule deux emplois, employé du ministère des Finances et ouvrier typographe dans une imprimerie, Mercedes/ Margarida, femme au foyer, coud des ourlets de pantalons pour arrondir les fins de mois avec l'aide de sa mère dona Herminia; l'aînée de la fratrie, Inés/Isabel travaille dans un salon de coiffure, Toni est sur le point d'entrer à l'université pour entreprendre des études de droit tandis que "Carlitos" qui est encore à l'école primaire, raconte l'histoire en voix off, une fois devenu adulte. Au fil des épisodes, on voit donc nos deux familles affronter les mêmes intrigues et accéder au confort de la modernité technologique proposé par la société de consommation.

Dans Cuéntame et Conta-me, l'Histoire contemporaine de la péninsule ibérique est, par conséquent, mise en fiction, replaçant l'histoire personnelle des Alcántara ou des Lopes dans le temps long. Le contexte historique des deux pays est constamment évoqué, d'abord à travers les informations radiophoniques qu'écoutent les différents personnages, puis grâce à la télévision qui arrive dans les deux foyers et devient un personnage à part entière. Les deux programmes instaurent, à travers la télévision que 
regardent les personnages, un dialogue continu entre le passé proche et le temps présent, un dialogue entre histoire et mémoire. Ce faisant, ils mettent à mal la frontière entre l'Histoire, conçue comme discipline scientifique de la connaissance des faits historiques et les œuvres d'imagination relevant de la fiction. Cuéntame cómo pasó et Conta-me como foi participent ainsi à leur manière du «vieux débat: celui de la confrontation entre le récit romanesque et le récit historique, en compétition pour dire le vrai d'une époque ", pour citer Mona Ozouf ${ }^{15}$. Cuéntame a même été présentée au public comme une série télévisée pouvant se définir par le triptyque formulé par Isabelle Veyrat-Masson dans ses travaux sur la dialectique existant entre Histoire et petit écran : «informer, cultiver, distraire ${ }^{16}$ ». Bénéficiant de moyens de production souvent réservés au cinéma, Cuéntame et Conta-me posent en sourdine la question de savoir si l'on peut exiger d'une série télévisée la même rigueur historique qu'un documentaire.

D’emblée placée à la croisée du divertissement et de la culture, la série espagnole a pour ambition de «contar » (raconter) le passé proche de l'Espagne avec la « rigurosidad histórica » (la rigueur des historiens) ${ }^{17}$. D'ailleurs, qu'il s'agisse de la version originale ou de son adaptation lusitanienne, les producteurs n'ont pas hésité à s'adjoindre les services de conseillers historiques spécialistes des périodes retracées pour s'assurer de la crédibilité de leurs propos : Laura Pousa ${ }^{18}$ pour Cuéntame et Helena Matos pour Conta$m e^{19}$. C'est également ce qu'insinue le slogan publicitaire de l'avatar original: "la historia de una familia española, la historia de todos " ("L'histoire d'une famille espagnole, l'histoire de tous ») qui a été repris dans tous les pays qui ont racheté les droits de la série. Par conséquent, les histoires que nous racontent les «Carlitos» seraient en fait celles de l'Espagne et du Portugal contemporains, une « histoire populaire » qui n'est certes pas académique mais prétend retracer le point de vue des anonymes qui ne figurent pas dans les manuels d'histoire.

10 La série a été vendue au grand public comme un docu-fiction en plusieurs épisodes dans lequel le souci des connaissances historiques et le soin apporté aux décors cœxisteraient avec la recherche de l'émotion et du divertissement. Tant Cuéntame que Conta-me s'appuient sur de nombreuses sources d'époque, qu'elles intègrent à leur trame narrative, tels que des films amateurs, des reportages, des spots publicitaires, des émissions de variétés, des séries télévisées ou des enregistrements audios, issus des fonds archivistiques de la RTVE ou de la RTP. Les différents procédés techniques utilisés par les réalisateurs (incrustation des personnages dans des images d'archives et autres documents d'époque) confèrent alors le charme désuet des documents historiques, tout en favorisant l'empathie des spectateurs avec les membres de la famille Alcántara et Lopes. Nos deux séries se présentent alors comme des fictions historiques c'est-à-dire qu'à l'image du "roman historique », elles visent à la représentation fictionnelle du passé effectif ${ }^{20}$. Toutefois, ni le soin apporté aux décors et aux choix des costumes d'une authenticité remarquable - ni les recommandations prodiguées par les conseillères historiques n'ont pu leur épargner de nombreux anachronismes ni de verser dans un certain simplisme. Soyons clairs, il ne sera pas question ici de dresser une liste exhaustive des erreurs que nous avons pu repérer dans les deux séries, mais de comprendre dans quelle mesure celles-ci ont bien constituées un choix délibéré de la production et d'en sonder les conséquences.

11 Un tel choix se voit légitimé par le fait que tant Cuéntame que Conta-me mettent en scène les conflits domestiques d'une famille tenue pour archétypale à la fin des années 
soixante, à travers le regard d'un enfant de 8 ans. Dans les deux cas, en effet, la narration est confiée à la voix de "Carlitos» en utilisant le «je " narratif. Les évènements sont rapportés et commentés en voix off par les narrateurs adultes dans chacun des épisodes. Loin d'être anodin, ce choix permet avant tout d'exploiter aisément le mythe de l'innocence enfantine. Les auteurs utilisent l'artifice du narrateur- enfant pour insinuer qu'ils posent un regard dépourvu de préjugés sur « un passé qui ne passe pas », pour reprendre une formule d'Henry Rousso. Quel discours ont alors voulu nous transmettre les showrunners sur les évènements rapportés par nos «Carlitos»?

En s'adressant directement au téléspectateur en des termes doux, naïfs et jamais violents, le récit des «Carlitos " verse dans la nostalgie. Les quelques timides critiques émises à l'encontre des régimes politiques en place sont d'emblée neutralisées. L'un des objectifs de cet article sera, dès lors, de mettre en lumière les intentions à l'œuvre derrière l'emploi d'un tel artifice rhétorique. Il nous faudra observer comment les auteurs mettent leurs personnages au service d'un discours idéologico-politique sur les transitions démocratiques ibériques. Nous chercherons à montrer comment le narrateur-adulte intervient sur la portée morale des séries. On constate qu'un télescopage a savamment été orchestré entre la mémoire individuelle, biographique, des deux Carlos et la "mémoire collective » qui articule le passé commun des membres composant la "communauté nationale » supposément représentée par l'ensemble des téléspectateurs pour susciter l'adhésion au récit généré. Les auteurs ont articulé les souvenirs des deux "Carlitos" de façon à ce qu'ils soient perçus comme des métaphores de la «mémoire collective » nationale. La question identitaire se trouve, par conséquent, placée au cœur de la trame narrative de Cuéntame et Conta-me. Bien plus, en adoptant un tel procédé narratif, il s'est agi de montrer que les Alcántara et les Lopes constituent, en quelque sorte, des idéaux-types de "l'hispanité » ou de la " portugalité » qui sont parvenus à gravir tous les échelons de la hiérarchie sociale à force de pugnacité et d'abnégation. Ce faisant ces séries participent non à l'écriture de l'histoire mais bien à celle d'un «roman national», expression popularisée par l'historien Pierre Nora dans ses fameux Lieux de mémoire qui évoque un

« récit patriotique, centralisateur, édifié par les historiens du xix ${ }^{\mathrm{e}}$ siècle tout à la louange de la construction de la nation. Le récit national met en avant la grandeur du pays, ses hauts faits et édulcore souvent les pages plus délicates. Il naturalise le "patriotisme" $" 1$ ".

13 À cause de leur succès, Cuéntame et Conta-me sont devenues des outils pédagogiques dans les collèges et les lycées pour l'apprentissage des langues et cultures ibériques, configurant dès lors l'appréhension de l'histoire des dictatures ibériques ${ }^{22}$. Elles modèlent l'imaginaire que l'on se forge sur cette période bien davantage que la lecture d'un ouvrage scientifique sur le sujet. Il s'agit donc pour nous d'analyser en profondeur le message transmis aux téléspectateurs à propos des transitions démocratiques. À travers le regard de "Carlitos", les réalisateurs proposent une vision inoffensive et anachronique du passé dictatorial des deux pays de la Péninsule ibérique, ces derniers étant présentés comme fondamentalement plus modernes et, surtout, plus européens qu'ils ne l'étaient en réalité. Ils nous donnent à voir une reconstitution des dernières années de dictatures hors-sol, une histoire mythique délibérément située dans la mémoire, c'est-à-dire dans l'émotion et en dehors de la sécheresse de l'Histoire.

Pour atteindre cet objectif, nous avons choisi d'articuler notre étude autour de deux principaux axes: dans un premier mouvement, nous procéderons à l'analyse des 
mécanismes de narration (le regard des enfants, les possibilités offertes par l'intertextualité et la place centrale tenue par la télévision dans le récit) pour, dans un dernier moment, nous pencher sur les représentations de l'Histoire sous-jacentes, les récits proposés sur les processus de transition démocratique. Nous tenterons ainsi d'appréhender les enjeux du récit mémoriel inhérents aux souvenirs évoqués par ces jeunes garçons de 8 ans, souvenir tout entier pétris de «saudade».

\section{Cuéntame cómo pasó et Conta-me como foi : le monde vu à la hauteur d'un enfant de 8 ans}

Cuéntame cómo pasó et Conta-me como foi sont des séries fondées sur un récit qui évoque plusieurs genres narratifs. Récit rétrospectif d'une enfance fait par un narrateur adulte qui intervient dans chaque épisode pour commenter ce que le téléspectateur vient de voir. Récit qui semble relever de l'(auto)biographique, s'appuyant sur des faits historiques avérés, des lieux et personnages réels. Mais, le récit raconté est bien une fiction puisque le narrateur n'est pas le scénariste et que par-delà des récits d'une vie, ces séries présentent certains aspects relevant de la magie et du merveilleux, éléments typiques des contes ou des fables. Deux récits simultanés se développent, dès lors, sur un événement vécu en commun, pour traduire deux points de vue.

\section{A - Deux narrateurs pour un même récit}

Cuéntame cómo pasó et Conta-me como foi sont des récits énoncés à la première personne du singulier, dans lesquels le narrateur - le petit «Carlitos» devenu Carlos une fois adulte - est aussi le personnage principal. Tous les épisodes sont introduits et conclus par la voix hors-champ de Carlos, le benjamin des trois enfants Alcántara et Lopes qui s'adresse aux téléspectateurs depuis l'époque actuelle avec sa voix d'adulte. Dans Cuéntame et Conta-me, les aventures quotidiennes des deux "Carlitos» constituent, ainsi, le fil conducteur de la diégèse sur lequel a été greffée une structure composée de cercles concentriques. D'après la chercheuse Charo Lacalle Zalduendo, professeur à l'Université de Barcelone et spécialiste des fictions télévisées catalanes :

«Cette structure narrative s'articule en trois cercles concentriques: la trame sentimentale, qui tient lieu de fil conducteur de l'histoire; les événements politiques et sociaux, dont la fonction est de doter chaque chapitre d'un référent, et les petits événements quotidiens touchant les personnages, qui servent essentiellement à récupérer la mémoire sociale collective de l'époque remémorée. Le résultat est un récit de fiction qui offre une reconstruction amène et didactique de la mémoire historique: la vraisemblance de ce récit est due à l'articulation pertinente des différents éléments qui le composent ${ }^{23}$ ».

Plus précisément, dans les deux séries étudiées, les narrateurs-adultes sont externes et racontent leurs souvenirs heureux d'enfance: c'est ce que Lacalle désigne sous la formule "trame sentimentale». Les narrateur-adultes séparent les différents événements ou moments rapportés en autant de séquences que la durée d'un épisode isole. Chaque épisode est considéré comme un « capitulo » c'est-à-dire " chapitre ", ainsi qu'il convient à un récit chronologique constitué à partir de souvenirs. Les récits possèdent cette particularité d'avoir été élaborés à travers les yeux, la pensée d'un jeune narrateur. Le point de vue reste interne, ce qui permet aux téléspectateurs d'entrer dans la conscience du personnage afin de mettre en place un lien privilégié 
entre eux. La période de la transition démocratique et ses conséquences tant en Espagne qu'au Portugal sont donc vécues de l'intérieur, avec la fraîcheur et «l'innocence » d'un enfant, ce qui est censé préserver le récit de trop de politisation, dramatisation ou pathétique. Dans ce contexte, les interventions du Carlos adulte ont été conçues comme devant favoriser une analyse teintée de recul de ce qu'il a vécu, de comprendre, par exemple, le sentiment d'étrangeté qu'il éprouvait souvent face aux événements politiques, aux difficultés éprouvées par ses parents.

On apprend donc l'identité de ce narrateur-personnage dès les pilotes c'est-à-dire lors du premier épisode: son nom (Carlos), son âge (8 ans), sa religion (catholique), sa nationalité (espagnole ou portugaise), les différents membres composant sa famille (père, mère, grand-mère, sœur et frère aînés) et ses centres d'intérêt (la télévision, notamment la série américaine Le Fugitif, et les jeux organisés avec ses camarades). Les scénaristes ont placé dans la bouche de ce narrateur-enfant des propos qui, à bien des égards, s'avèrent caractéristique de cette tranche d'âge : Carlos ignore souvent certains termes et mélange les mots lorsqu'il cherche à décrire une réalité. Il ne comprend pas le sens figuré et s'arrête au sens littéral. Par exemple, il ne comprend pas la blague faite par Toni lors du dîner sur le dictateur Franco (version espagnole) ou la police politique (version portugaise) ${ }^{24}$. Il utilise une syntaxe oralisée marquée par la présence abusive de conjonctions de coordination. Le narrateur-enfant utilise de nombreuses phrases à présentatif et emploie un registre de langue populaire. Dans les deux versions, son ingénuité d'enfant de 8 ans est mise en avant lorsqu'il croit, par exemple, que les « Rouges » sont des Indiens ou qu'il prend l'illustration de la République que lui montre son frère pour Brigitte Bardot parce qu'elle a " una teta al aire », " uma mama ao ar » (" un téton à l'air ») ${ }^{25}$. L'humour est toujours présent derrière les remarques ou les inventions de Carlitos, comme lorsqu'il déclare «Franco es un prohibidor» ou "Salazar é um proibidor " parce qu'ils interdisent tous deux le divorce ${ }^{26}$, ou met dans une tombola le dentier du grand-père d'un de ses camarades en le faisant passer pour celui du dictateur ${ }^{27}$. Comme tous les enfants, le narrateur s'interroge constamment, il essaie tant bien que mal de comprendre les événements politiques ou le sens des mots " colonialisme », « impérialisme ", « dictature » et le sens des mots qui composent leurs définitions. Sa langue contient également des confusions sémantiques ${ }^{28}$ et Carlos déforme sans le vouloir autant des expressions lexicalisées célèbres que les leitmotivs du régime autoritaire.

19 À côté de "Carlitos ", on observe, ensuite, le véritable narrateur: le même Carlos devenu adulte. Dès les premières minutes de l'épisode 1 , le narrateur se présente comme l'auteur du récit et annonce que près de quarante années se sont écoulées depuis les faits que lui-même vient de rapporter. À l'intérieur même du récit rétrospectif, ce narrateur-adulte intervient à de nombreuses reprises dans le déroulement de tous les épisodes. Dès les premières phrases prononcées dans le pilote, le récit est mis dans la perspective de l'adulte penché sur son passé. Cuéntame et Contame adoptent ainsi le point de vue d'un adulte, présent au seuil de chaque récit et parfois aussi en son cœur, grâce à la voie hors-champ. L'adulte prend le relais de l'enfant quand il faut expliquer des faits que celui-ci ne pouvait connaitre et comprendre pleinement à l'époque, tels que les dangers accrus de « parler de politique » chez soi car « les murs ont des oreilles ». Le narrateur-adulte intervient d'abord pour cadrer le récit dans la chronologie de la période évoquée, la transition démocratique étant une notion floue pour un jeune enfant, et résumer certaines périodes clef de sa vie. Le narrateur- 
adulte prime donc sur le narrateur-enfant puisqu'il est le " personnage point de vue " commentant, a posteriori, les actions des personnages. Le ton amusé de certains passages donne à voir le narrateur-adulte face à l'enfant qu'il fut, pour son incompréhension de ce qu'ont été la dictature, la répression ou la censure ou dans ses descriptions des personnages qu'il a rencontré et dont un trait l'a profondément marqué comme le doigt "suspect» de l'épouse française de son oncle paternel qui a émigré en France et qu'il considère être une "preuve » du fait qu'elle est forcément une extraterrestre, un "envahisseur" camouflé venu en éclaireur ${ }^{29}$. Il explique également ses propres sentiments de façon rétrospective : son manque d'attirance pour les filles de son quartier qui sont autant d'ennemies à combattre, ou ses joies et déceptions partagées avec ses camarades de jeu. Par ailleurs, il éclaire le récit de la sagesse gagnée au fil des événements ou émotions de son enfance, utilisant pour ce faire le présent de vérité générale comme lorsqu'il déplore l'absence de terrains vagues pour jouer du fait de l'urbanisation massive, du manque d'espaces verts dans les grandes villes d'aujourd'hui ${ }^{30}$. L'adoption du point de vue des narrateurs-adultes entraîne, par conséquent, le regard nostalgique sur l'époque décrite qui, pour terrible qu'elle ait pu être, reste dans sa mémoire (sélective et déformante par définition), celle de l'enfance et de l'insouciance.

La dialectique entre le narrateur-enfant et le narrateur-adulte montre que la composition de ces séries est significative: les scènes initiales et finales de chaque épisode attestent que, bien plus qu'un récit autobiographique d'un enfant ayant grandi sous un régime national-catholique autoritaire, il s'agit d'un parcours spirituel, moral qui est, devant les téléspectateurs, retracé. Le personnage de Carlos concentre sur lui la focalisation du récit. Il est un « point d'entrée » dans la fiction, un support privilégié de l'empathie. Le narrateur, à l'occasion des dernières années de la dictature au cours desquelles il n'a pas vraiment souffert, a vécu une expérience intérieure qui porte ses fruits quarante années plus tard. En cherchant à se remémorer les bons moments de son enfance, le narrateur devient lui-même, au fil des épisodes, dépositaire et relais d'une mémoire pour les générations futures.

21 Ainsi, tant dans Cuéntame que Conta-me, le récit se rapproche ainsi du biographique par plusieurs aspects : d'abord, il se donne comme le récit d'une vie, et n'était la différence de nom entre le personnage principal et ceux des scénaristes présents au générique, on pourrait tout à fait le croire autobiographique. Ensuite, ce récit est inscrit dans la réalité de différentes façons : par les noms des dirigeants politiques qui se réfèrent à des personnalités historiques (Franco, Salazar, Caetano, etc.) ; par les reconstitutions soignées des décors et lieux qui donnent à voir le contexte de l'époque retracée. Les deux séries traitent d'événements décrits dans les livres d'histoire. Les faits historiques marquants de la période sont évoqués par l'entremise des unes de journaux, les actualités télévisées ou, tout simplement, à travers les propos "café-du-commerce " tenus en sourdine entre voisins au kiosque du marchand de journaux ou dans la taverne du quartier. Elles sont très documentées et, au premier abord, on pourrait penser qu'elles sont relativement fidèles à la réalité historique. La chercheuse Susana Diaz, en analysant Cuéntame, écrit que la « vérité historique » est connue

« à travers certains décors, costumes (...) grâce à la récupération des tics linguistiques et expressions idiomatiques de l'époque (...) références constantes aux produits de consommation emblématiques (...) et des citations continues des produits de la culture populaire ${ }^{31} »$. 
Les reconstitutions permettent ipso facto d'installer les téléspectateurs dans le familier et le commode du passé proche. Les auteurs de Cuéntame et Conta-me ont pu axer leurs propos sur l'authenticité, la vraisemblance. Gérard Genette, dans Figures II, affirme que le vraisemblable s'identifie bien souvent au « devant être » c'est-à-dire ce qui s'avère être conforme à la doxa - l'opinion publique - et fonctionne comme une «maxime du monde $~^{32}$. Les producteurs ont pris le parti d'une esthétique fondamentalement réaliste en focalisant la majeure partie des intrigues sur les difficultés quotidiennes des habitants de San Genaro et du "bairro social " lisboète sous les dernières années des régimes dictatoriaux ibériques. Pour reprendre l'analyse forgée par Roland Barthes, la mimèsis ou « l'effet de réel (...) vise l'adhésion du lecteur à la réalité du récit; l'effet de fiction nous plonge dans l'univers d'une non-réalité ou d'une réalité autre, celle de la fiction $^{33}$ ». Cuéntame et Conta-me exploitent, par conséquent, la confusion existant entre les programmes de téléréalité qui émergent au même moment sur le petit écran pour

«mett[re] en scène le banal, le quotidien, et qui prône[nt] comme valeur fondatrice

l'absolue transparence, le complet effacement du medium (...) prétend[a]nt donner accès à la réalité de l'expérience de leurs protagonistes ${ }^{34}$ »

et le soap " vou[é] à la représentation de l'intime et du familier, à la monstration du banal $»^{35}$. La distinction entre ces deux types de programmes résident dans leur degré de « narrativisation ». "La téléréalité, écrit encore Marine Legagneur, donnait accès à un quotidien pour partie fictionnel de personnages réels, le soap donnera donc accès au quotidien réaliste de personnages de fiction ${ }^{36} »$. Pareille confusion suggère que les faits évoqués sont certes fictifs mais auraient très bien pu se produire tel quel dans la réalité historique.

\section{B - Conta-me como foi : intertextualité et « transadaptation »}

Conta-me como foi apparaît comme une "réécriture » de Cuéntame cómo pasó en ce sens que les scénaristes de la version portugaise ont «donn[é] une nouvelle version d'un texte déjà écrit ", ils ont " réinvent[é], donn[é] une nouvelle version de quelque chose ${ }^{37}$ ». Pour paraphraser G. Genette dans Palimpsestes, il existe entre ces deux séries « une relation manifeste ${ }^{38}$ ", une intertextualité affirmée dans la représentation du passé proche. Cette intertextualité apparait à deux niveaux. D'abord, comme dans la version originale, le petit "Carlitos" est fasciné par ce que diffuse le poste de télévision nouvellement arrivé dans son salon et fait souvent référence à des programmes ou séries appartenant à la mémoire collective. Pour concevoir les jeux avec ses camarades, il n'est donc pas rare que Carlitos s'inspire du déroulement d'un épisode d'une série Le Fugitif ou Les Envahisseurs - pour décider de leur activité. Cette intertextualité est transparente, en ce sens que les téléspectateurs (même les plus jeunes qui n'ont jamais vu le programme ou la série cités) ne peuvent manquer la référence intertextuelle. C'est ce phénomène que Séverine Barthes, dans sa thèse de doctorat, désigne comme "des citations marquées ${ }^{39}$ ". Ces dernières peuvent également être visuelles: les affiches de films accrochées aux murs, les publicités diffusées à la télévision, les produits ménagers ou alimentaires de l'époque posés en évidence sur la table du salon ou de la cuisine... Ensuite, le second niveau de l'intertextualité est ce que l'autrice qualifie de "phénomènes de références ». Ils sont toutefois moins explicites que les "citations marquées" dans la mesure où la citation n'est perçue que par les téléspectateurs qui connaissent l'intertexte de la version originale espagnole. Dès lors, on retrouve souvent dans Conta-me les mêmes situations, mises en scène, plans ou 
travellings que dans Cuéntame. Le mimétisme a été poussé jusqu'à donner des titres identiques à plusieurs épisodes. Dans le pilote, par exemple, la voix adulte de Carlos Lopes, en off, prononce les mêmes commentaires que ceux de Carlos Alcántara dans le même épisode de Cuéntame cómo pasó :

Carlos Lopes: «Em 1968 eu tinha oito anos. Às vezes as pessoas dizem que o ano de 68 foi o principio da mudanza, mais foi pelo menos para mim. A verdade é que em $1968 \mathrm{eu}$ non reparava nas coisas importantes que mudavam á minha volta. Tinha outras preocupaçœs. Preocupava-me, por exemplo, com o destino do Fuxitivo... ».

Carlos Alcántara: "En 1968 yo tenía 8 años, (ahora dicen que en 1968 fue un año revolucionario y lo fue por lo menos para mí). La verdade es que en esa época yo no estaba para revoluciones me preocupaban otras cosas, por ejemplo, que el pobre Fugitivo... $»^{40}$.

Ces phénomènes de "référence » ou "citations masquées » irriguent tous les pans de Conta-me como foi. Toutefois, celle-ci s'est vue contrainte de transposer les enjeux et éléments culturels typiquement espagnols de la série originale pour les rendre conformes au profil et aux attentes des téléspectateurs portugais. Pour renforcer la crédibilité du scénario et susciter l'adhésion, il est nécessaire d'élaborer un récit " qui entre en résonance avec l'expérience spectatorielle $»^{41}$, il a donc été nécessaire de les " portugaliser » c'est-à-dire que les intrigues et enjeux ont été adaptés, transposés aux particularismes, préoccupations et traditions locaux. Les bandes sons, les images d'archives et autres références utilisées permettent de mettre en scène le patrimoine culturel et populaire du pays dans lequel nos séries s'enracinent. C'est ce que la sociologue italienne travaillant sur la culture populaire Milly Buonanno appelle des "transadaptations ${ }^{42}$ ». Toute importation de format pour rencontrer le succès, souligne-t-elle, doit inévitablement être "transposée » à l'aune d'une identité locale. Les «transadaptations", poursuit-elle, ne peuvent alors être possibles que s'il existe une " proximité culturelle ${ }^{43}$ » entre le pays créateur du format et celui qui l'importe sur son marché. La "transadaptation» concerne évidemment toutes les formes de transferts linguistiques et culturels afin de faciliter la plausibilité du récit raconté. On ne traduit pas littéralement les dialogues si cela ne permet pas la cohérence de la narration mais les textes sont traduits en fonction d'un contexte audio et visuel qui impose, par conséquent, tout un ensemble de règles et conventions à respecter.

\section{Un exemple tangible de " transadaptation » : "Educación y mundología » (S01E11) de Cuéntame et "Do Minho a Timor " (S01E12) de Conta-me}

L'épisode 12 de Conta-me como foi intitulé « Do Minho a Timor » constitue un bon exemple de «transadaptation" de l'épisode 11 de la version espagnole originale intitulé « Educación y mundología ». Comme on le montrera par la suite, les similitudes entre " Educación y mundología » et "Do Minho a Timor » sont particulièrement frappantes. Le décalage observé dans la chronologie des épisodes peut aisément s'expliquer par les différents contextes historiques des pays ibères. Les showrunners n'ont choisi que de «transadapter » certains épisodes dans la masse que comptait déjà Cuéntame en 2007. De plus, durant la première saison de la version portugaise, un épisode a été ajouté par rapport à la version originale afin de traiter de l'accident cérébral dont a souffert le président du Conseil Salazar après avoir chuté de sa chaise ${ }^{44}$. Toutefois, dans les deux épisodes analysés ici, la trame narrative est restée identique: António, le père de famille, craint de perdre son travail d'appoint à l'imprimerie depuis que son patron a décidé de moderniser son négoce en acquérant une machine dernier cri de "qualité allemande » qu'il ne parvient pas à faire fonctionner. En bon pater familias, il refuse de 
voir son épouse travailler en dehors de la maison car, pétri de machisme, il est effrayé par la perspective qu'elle puisse gagner plus d'argent que lui en devenant autoentrepreneuse et cumulant les contrats. Inés/Isabel, l'aînée de la fratrie, ne sait pas si elle doit ou non se marier et éprouve un sentiment d'injustice profonde dans une société qui discrimine les femmes en les empêchant de poursuivre leurs études ou voit d'un mauvais œil le fait qu'elles tiennent un commerce seules par exemple. Quant à Toni, il cherche à s'impliquer davantage dans la lutte contre le régime qui met en ébullition le milieu estudiantin, mais peine à comprendre la différence entre les nombreux groupes politiques ou pourquoi ils contestent en premier lieu l'intervention américaine au Vietnam.

Cependant, toutes ces préoccupations des adultes paraissent insipides à côté des aventures que vivent Carlos et ses amis, les garçons se retrouvant constamment impliqués dans des situations farfelues, ubuesques. Le point nodal réside dans le fait que dans ces deux épisodes, les enfants s'approprient le discours impérialiste de l'Estado Nueve franquiste/Estado Novo salazariste mais ne parviennent pas à en saisir toute la portée. De leur mésinterprétation et volonté de soutenir le régime naît un comique de situation particulier: le quiproquo. Quiproquo qui les conduira d'ailleurs en garde-àvue. À partir de cette trame narrative, il a, dès lors, fallu adapter les principaux éléments de l'intrigue au cadre spatial portugais et veiller à prendre en considération les particularités locales sous-jacentes. L'épisode «Educación y mundología » est composé de trois séquences qu'il a fallu « transadapter » au contexte portugais.

\section{Séquence 1 : La soirée familiale}

La version originale s'ouvre sur la famille à table. Pendant le repas, Toni, tente de faire une blague sur le dictateur. Toutefois, même s'ils sont dans l'enceinte privée de leur appartement, la peur que « les murs aient des oreilles » pour reprendre une expression populaire et d'être dénoncés par un voisin malveillant fait qu'Antonio enjoint à son fils de ne pas plaisanter avec ces choses-là. On le voit, dès l'ouverture de l'épisode, le régime est une source constante de peur pour l'ensemble des protagonistes. Carlos et les femmes de la famille insistant pour entendre la blague, le patriarche consent à écouter son fils.

«Antonio Alcántara : No será de Franco.

Toni : Que no papa, no es de Franco.

Antonio : Ya sabes que no me gusta que te vayas aí contar chistes de Franco.

Herminia : Haz caso a tu padre que tiene razón.

Toni : Pero si solo lo cuento en casa.

Herminia : Pero ¿Y si te oyen los vecinos?

Toni : Pero, lo cuento en bajito.

Carlos : Papá, déjale.

Antonio : Es que es un bocazas... lo cuenta por ahí y no sabe lo que puede pasar.

Mercedes : Anda, António, es solo un chiste.

Antonio : Bueno, cuenta el chiste pero cuenta lo bien.

Toni : Esto es un pueblo perdido y llega al alcalde un telegrama de Madrid. El alcalde, emocionadito, porque no está acostumbrado que le llegue un telegrama de Madrid, lo lee y dice: "Movimiento sísmico detectado en su zona. Localicen epicentro PUNTO".

Carlos: ¿Qué?

Herminia : Qué palabras más rares usa este niño.

Mercedes : Deja que acabe.

Carlos: Es que no lo entiendo nada. 
Antonio : Calla, hijo.

Toni : El alcalde, el pobre hombre que no entiende nada de lo que dice.

Carlos : Toma, ni yo.

Mercedes : Calla.

Toni : Total que al final dice : Que venga el cabo de la Guardia Civil. Y llega el cabo de la Guardia Civil, con su uniforme y su bigotito, y le dice : Usted tranquilo, esto lo soluciono en un momento. Le vamos a mandar un telegrama de respuesta a Madrid que diga : "Movimiento sísmico sofocado. Epicentro y dos más detenidos" « ${ }^{45}$. a, quant à elle, dû subir quelques transformations pour la rendre cohérente avec la réalité portugaise : la Guardia Civil espagnole a été remplacée par la police politique portugaise, la PIDE et, bien évidemment, la figure de Franco est devenue Salazar. Il a même été spécifié que le vieux président du Conseil était malade.

Une fois que son fils a terminé de raconter, Antonio peinant à comprendre ce qu'il vient d'entendre doit feindre une quinte de toux pour aller, en catimini, chercher dans un dictionnaire le sens du terme "sismique». Il revient ensuite à table en riant allègrement et, devant l'incompréhension générale et celle de son plus jeune fils en particulier, il leur explique le sens de la blague. Carlitos se met enfin à rire et affirme que son père «sait tout sur tout». La voix off de Carlos adulte intervient alors pour conclure la séquence :

«cuando yo era pequeño estava convencido que mi padre era el hombre más sabio de la tierra. No solo tenía las respuestas sino que las decía con una autoridad que ni sequer mis maestros podía emular. Tener un padre así era un lujo ${ }^{46}$ ”.

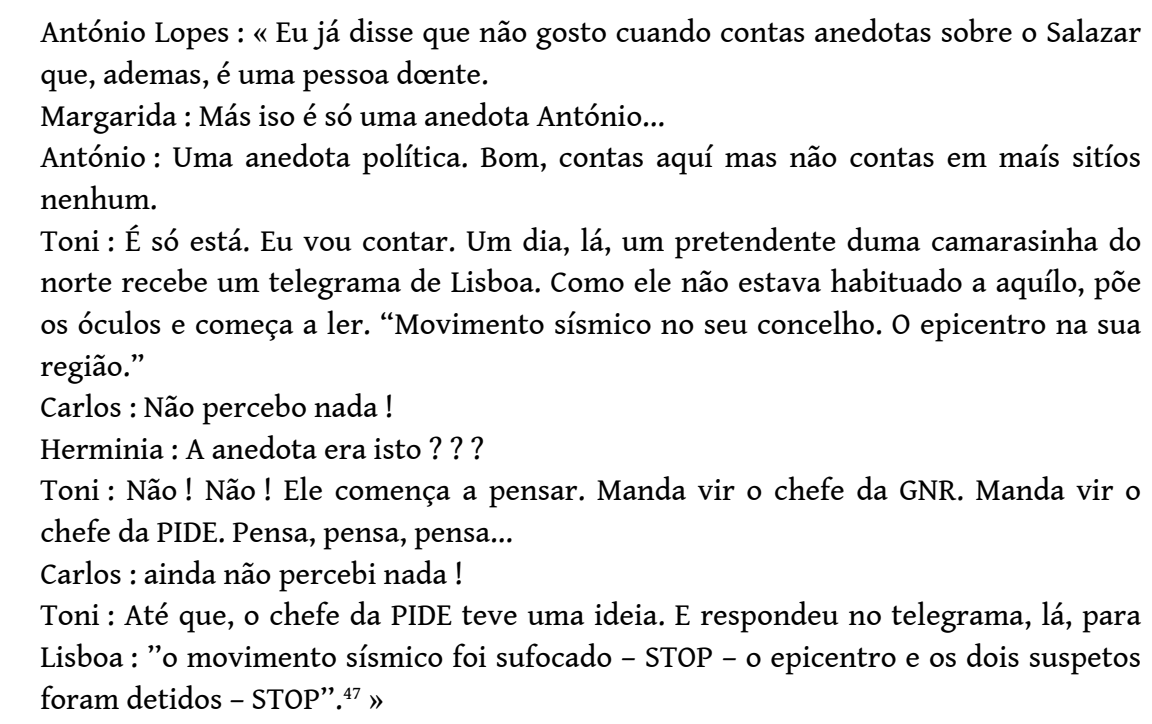

Le même comique de situation est repris. António doit, lui aussi, feindre une quinte de toux pour aller discrètement feuilleter un dictionnaire encyclopédique et trouver le sens du terme « sismique ». Une fois la blague comprise, il revient au salon et devant la figure pantoise de Carlitos, il se met à expliquer que le chef de la PIDE voulait en fait emprisonner le tremblement de terre ainsi que deux autres suspects. Content d'avoir enfin saisi la portée du mystère, le garçonnet s'exclame que son "père sait tout ». La scène se termine avec l'intervention du narrateur-adulte qui, comme son homologue espagnol, affirme, un trémolo de nostalgie dans la voix,

«Cuando eu era pequeno, acreditava piamente que o meu pai era o homem mais sábio do mundo. Não só tinha resposta para todas as perguntas como, ainda por 
cima, as debitavas com uma autoridade que nem o professor Rui Braga conseguia

ter. Ter um paí asim tão sabio era um luxo e um orgulho ${ }^{48} »$.

31 sur Gibraltar, ancienne colonie, insistant fortement sur l'idée que « les Anglais [leur] ont volé ce trésor national». Il y est spécifié que sur le poste de douane situé à la frontière flotte désormais « un drapeau noir de la séparation ». Toni intervient alors pour dire que les «Yankees» sont pires que les Britanniques et qu'il envisage de participer à une manifestation contre la "guerre impérialiste» menée par les Américains au Vietnam. Quant à l'épisode portugais, le cadre intime a été préservé mais la toile de fond a dû être modifiée, adaptée pour coller à la réalité de l'époque. Le Portugal sous l'Estado Novo était, en effet, profondément isolé sur la scène internationale, l'ONU et l'OCDE ayant adopté plusieurs résolutions et un embargo à son encontre pour condamner son attitude jusqu'au-boutiste dans la poursuite des guerres coloniales en Afrique. Depuis 1961, le pays vit replié sur lui-même et son empire, préférant tourner le dos à la Communauté internationale plutôt que d'abandonner " 0 que é nosso $»^{50}$. Le président du Conseil Salazar affirmait d'ailleurs en novembre 1965 que les Portugais combattaient en Afrique "sem espetáculo e sem alianças. Orgulhosamente, sós $"^{51}$ contre l'hydre communiste. Le Portugal consacrait près de $50 \%$ de son budget national au maintien par la force de son empire. Pour justifier une telle dépense, la propagande avançait le discours véhiculé par le reportage/documentaire regardé par les Lopes selon lequel «le Portugal n'est pas un petit pays» mais un "Empire pluricontinental » s'étendant " do Minho a Timor » et pouvant rivaliser tant en superficie qu'en populations avec les deux superpuissances mondiales que sont l'URSS et les ÉtatsUnis. Par conséquent, dans l'épisode portugais, il n'est point question de "guerre impérialiste américaine» ni de drapeau étoilé brûlé sur un campus en signe de protestation contre le soutien implicite qu'apporte la démocratie américaine à Franco en s'accommodant parfaitement de la nature autoritaire de son régime. Il ne sera pas non plus question d'étudiants - parmi lesquels se trouve Toni - fuyant devant la Guarda civil. Dans la version lusitanienne, il s'agira de s'insurger contre les troupes indiennes et les Espagnols. Pour s'adapter au contexte local, en effet, le documentaire propagandiste sur Gibraltar a été remplacé par un propos sur la perte en 1961 du contrôle de ses colonies en Inde - Goa, Daman et Diu - à la suite d'une "annexion» par l'armée indienne. Le film qui, se veut pédagogique, affirme que cet acte constitue un « affront à l'intégrité nationale», un acte "totalement illégitime». À l'instar du petit Carlos Alcántara, Carlitos Lopes veut donc savoir pourquoi le Portugal a été spolié de ses colonies. Et, là-encore, c'est Toni qui s'évertue à expliquer à son petit frère les mensonges colonialistes du régime. Cependant, au lieu de s'en prendre à "l'impérialisme yankee », il s'en prend ouvertement à l'impérialisme lusitanien en proclamant, non sans ironie, "Quem é que roubou quem, Carlitos? Foram os indianos, ou foram os portugueses? " $^{52}$.

\section{Séquence 2 : Dans la salle de classe}

Dans les deux versions, vient ensuite une scène tournée dans la salle de classe de Carlos. Dans Cuéntame, son professeur - une caricature nationaliste en soi - donne une leçon sur le traité d'Utrecht qui céda aux Anglais le contrôle sur Gibraltar. Ému par les vociférations de son instituteur, Carlos décide alors de devenir un héros national et d'entreprendre une croisade pour aller réclamer la rétrocession de Gibraltar à 
l'Espagne. Après la classe, une fois parvenus au terrain vague, lui et ses amis planifient la reconquête du Peñón et s'entraînent au maniement des armes (en bois) pour être capables d'affronter sur le champ de bataille les troupes anglaises. Après cette journée riche en émotions, le petit Carlitos se rêve sous les traits d'un guerillero madrilène martyr, les bras en croix avec les marques de la crucifixion sur les mains, s'apprêtant à être fusillé par l'ennemi. La mise en scène est le décalque du célèbre tableau $\mathrm{El}$ Tres de Mayo de Goya représentant l'exécution de 43 espagnols, fusillés par les soldats de «l'envahisseur français » à Madrid le 3 mai 1808, pendant la nuit. Bien évidemment, dans le contexte relaté ici, Carlos s'imagine combattre l'impérialisme anglais plutôt que français. Le jeune garçon se fantasme au cœur de ce tableau dans la mesure où cette œuvre représente la première révolte nationale en Europe contre l'impérialisme bonapartiste. Pour cette raison, il est devenu le symbole de l'indépendance et de l'identité nationale espagnole. Son souhait, comme il le disait à ses camarades, est de mourir en « héros de la patrie " pour être enterré dans le Valle de los Caídos.

Carlos Alcántara : «Es que así, si nos matan, seremos hérœs de la patria.

Josete : ¿No podemos ser hérœs vivos?

Carlos Alcántara: No, porque no nos enterrarían en el Valle de los Caídos. Ahora a

vigilar $^{53}$ »

Dans Conta-me, nous retrouvons Carlos en classe avec son enseignant totalement exalté par le discours nationaliste du régime. Depuis l'explosion de la guerre coloniale en 1961, le nationalisme est abondamment inoculé aux écoliers afin de leur apprendre à « aimer leur Empire ». L'adaptation aux couleurs locales a signifié que la leçon du jour porte sur le traité de Tordesillas ${ }^{54}$ et non Utrecht. L'instituteur relaie efficacement la propagande salazariste et rappelle que l'Espagne a toujours voulu intégrer le pays à ses possessions depuis la signature dudit traité. La Maison de Castille a même été jusqu'à l'annexer en 1580 pour constituer une " Union ibérique " sous l'égide de Felipe II après la disparition prématurée du roi D. Sebastião. Le Portugal n'est parvenu à recouvrer son indépendance qu'après la guerre de Restauration en 1640. Ce discours sur «l'appétit annexionniste castillan » a enthousiasmé le jeune Carlos qui cherche à comprendre pourquoi «O Espanhol robo Olivença ? $\|^{55}$. Comme dans Cuéntame, après la classe, Carlitos et ses comparses se retrouvent sur leur terrain vague et s'entrainent vaillamment pour reconquérir le territoire perdu contre l'ennemi. Ils s'imaginent sous les traits de preux chevaliers du Moyen Âge rejouant l'exploit militaire de la bataille d'Aljubarrota du 14 août 1385 où les Portugais parvinrent à assurer l'indépendance du royaume vis-à-vis de la Castille. Carlos Lopes et ses amis rêvent de mourir en martyrs pour la patrie et d'être enterrés au Mosteiro dos Jerónimos à Lisbonne plutôt qu'à Valle de los Caidos.

Carlos Lopes: Marinho tems que decidir entre a garagem da Lego e ser um herói. Os heróis já estam todos mortos más se morremos pela a patría vamos ser condecorados no 10 de junho [Jour du Portugal et de la Race] e talvês nos levam para os Jerônimos. Va, vamos começar a vigiar $!^{56} »$.

\section{Séquence 3 : La manifestation officielle}

La troisième scène transposée est celle de la manifestation. Dans Cuéntame, Cervantes, le vieux marchand de journaux du quartier, invite Carlos et ses amis à se joindre à lui lors d'une manifestation organisée par le gouvernement pour exiger la restitution de Gibraltar à l'Espagne.

«Carlos Alcántara : ¡Vamos a reconquistar Gilbraltar!

(...) 
Cervantès : Pero antes tenéis que ir a la manifestación del domingo.

Josete : ¿Qué manifestación?

Cervantès : La de Gibraltar español. Va a ir todo el mundo, y yo el primero ${ }^{57}$ ".

Après avoir discuté avec son frère aîné, Carlos qui, ne connaissait pas jusque-là la signification de ces termes, a appris que « l'impérialisme » est une mauvaise chose tout comme l'est la «dictature». Ainsi, lors de la manifestation contre "l'impérialisme anglais ", Carlos et ses amis ont commencé par crier "Abajo el imperialismo» et sont rapidement passés au slogan "Abajo la ditadura ». Bien entendu, les enfants n'ont pas conscience de la portée subversive de leur slogan, ils sont arrêtés et Antonio est convoqué au poste de police pour subir un interrogatoire avant que son fils ne soit libéré et rentre, sain et sauf, à la maison. Dans Conta-me, on retrouve le même schéma actanciel. Là-encore, la conversation qu'ont Carlos et ses amis avec Camões, le propriétaire du kiosque à journaux du quartier est le nœud de la trame narrative.

"Carlos Lopes: Vamos reconquistar Olivença aos Espanhóis! Eles não são pretos, pois não?

Camões : Não, são brancos por fora e amarelos por dentro. E o amarelo é a cor da traição. De

Espanha, nem bom vento, nem bom casamento $"^{58}$

En effet, à l'image de Cervantes, Camões invite les enfants à une manifestation organisée par le gouvernement pour protester contre la perte de Goa.

«Carlos Lopes : É uma manifestação contra os Espanhóis?

Camões : É como se fosse $!^{59}$ »

Armés de leur banderole sur laquelle on peut lire "Abaixo o Imperialismo", les enfants contaminés par l'euphorie collective se mettent à vociférer "abaixo a ditadura » et, là encore, parce qu'ils n'ont pas conscience de tenir des propos hautement subversifs à l'encontre des autorités publiques qui persistent à envoyer des contingents combattre dans les colonies africaines, ils sont arrêtés et emmenés au commissariat de police. Dans les deux versions, c'est par syllogisme - parce qu'ils associent les Anglais (Cuéntame) ou les Espagnols (Conta-me) à la méchanceté et la méchanceté à la dictature que les garçonnets ont poussé ce cri de revendication. Dans ces deux épisodes, Carlos s'apparente ainsi à une sorte de "résistant malgré lui ». Pendant que les enfants attendent que leurs parents viennent les récupérer, Carlos conçoit un plan d'évasion et se démène pour creuser un tunnel dans ce qu'il imagine être sa cellule. La «transadaptation" a exigé que Carlos Lopes soit persuadé que le policier qui les a surveillé était, en réalité, un agent espagnol infiltré...

On le voit, les deux épisodes analysés exposent la propagande impérialiste propre à chacun des régimes et la façon dont la population y a consenti. La version espagnole montre l'hypocrisie du gouvernement de Franco qui a décrié la présence anglaise à Gibraltar mais soutenu les troupes américaines au Vietnam. La version portugaise en évoquant la blessure d'orgueil qu'a constitué la perte de olivença rappelle, quant à elle, la manière dont le gouvernement de Marcelo Caetano instrumentalisait, comme jadis le faisait Salazar, le "péril espagnol» et la peur d'une annexion par l'Espagne phalangiste. Dans l'épisode portugais, le discours colonialiste ne relève toutefois pas simplement de la nostalgie sur la grandeur passée du pays mais bien davantage de la volonté de maintenir la présence portugaise en Angola, Guinée-Bissau, Mozambique, Cap-Vert et São Tomé e Principe. Contrairement au cas de Goa, celui d'olivenza relève bien comme celui du Gibraltar espagnol de la «Phantasie» théorisée par Freud désignant pour le psychanalyste « un scénario imaginaire dans lequel le sujet accomplit un désir inconscient». L'animosité portugaise envers l'Espagne a toujours été un puissant levier pour galvaniser les foules autour de la défense de «l'intégrité 
nationale » du Portugal et son Empire. L'Estado Nuevo de Franco avec sa Falange étaient perçus comme des instruments commodes par les autorités salazaristes pour justifier non seulement l'imposition d'un État d'exception et d'un droit dérogatoire pour «lutter contre le terrorisme » tant intérieur qu'extérieur mais également surveiller les frontières en vue de réduire l'émigration endémique qui vidait le pays de ses jeunes hommes ne voulant pas être enrégimentés ${ }^{60}$.

\section{C - La télévision dans Cuéntame et Conta-me como foi, un « effet d'enfance » ?}

39 L'intertextualité a été facilitée par le fait que la série ménage une place importante du point de vue narratif au support télévisuel. La télévision a savamment été intégrée à la diégèse jusqu'à, parfois, prendre temporairement en charge la narration. À l'image de nos deux Carlitos, plusieurs générations ont grandi en regardant le petit écran. À travers les images qu'il projette, les souvenirs individuels, particuliers, peuvent être rattachés à la réalité historique de l'époque que Carlos tente de nous faire revivre. Toutefois, fort paradoxalement, on constate que la télévision contribue surtout à la marginalisation des circonstances historiques. Dans nos séries, en effet, les programmes télévisés ne sont qu'un simple décorum, un prétexte pour raconter une saga familiale. L' «arrivée de l'étrange lucarne ${ }^{61}$ » dès les pilotes constitue le point de départ de la diégèse et, à compter du moment où l'antenne a été reliée, ce sont bien ses émissions qui impriment la temporalité du récit. L'arrière plan historique reste, par conséquent, assez imprécis, se résumant à ce qu'a pu diffuser la télévision: les feuilletons américains comme le Fugitif ou Les Envahisseurs, un mariage princier, l'arrivée du premier homme sur la lune, la première greffe cardiaque réalisée par Christian Barnard, le concours de l'Eurovision ou celui du Festival da Canção Portuguesa (Festival de la chanson portugaise), des galas mondains, des documentaires animaliers, des spots publicitaires, etc...

40 Le recyclage d'images d'archives - principalement des images audiovisuelles - par les scénaristes est très intéressant dans la mesure où cette pratique est une autre facette de l'intertextualité au sens proposé par Gérard Genette dans Palimpsestes. La présence d'images d'archives, un peu comme celles des illustrations dans les livres pour enfants, autorise une forme décalée d'intertextualité, ce qu'on peut désigner par une « intericonicité ${ }^{2}$ ». Clément Chéroux préfère, quant à lui, parler de "mobilisation d'images-palimpsestes». Pour lui, les images intericoniques "fonctionnent sur ce même principe [l'intertextualité] et renvoient autant, sinon plus, à d'autres images qu'à la réalité de l'évènement dont elles sont la trace directe ${ }^{63} »$. L'intericonicité propre à la reprise télévisuelle repose assurément sur un présupposé de connaissance préalable des images, permettant ainsi aux téléspectateurs de déceler l'emploi renouvelé de ces images, d'analyser ce « déjà-vu ${ }^{64}$ ». Le téléspectateur qui a lui-même été un enfant peut rêver sur les extraits de programmes et sa mémoire s'alimente à la nostalgie de ces émotions anciennes, à la primitive fascination des images.

41 Les chaînes de service public ont vu le jour en Espagne et au Portugal à la fin des années $1950^{65}$. Cette " ancienneté » signifie que l'incroyable masse d'archives tournées depuis a été une manne de documents authentiques, "déjà là » - à disposition - et " déjà vu » par le plus grand nombre, pour les réalisateurs. Ces derniers ont pu piocher dans ces fonds tous les éléments de contextualisation et exploiter efficacement le filon de la 
nostalgie. Un tel choix est assez compréhensible. La reprise d'extraits de programmes déjà diffusés (voire multi-rediffusés) a construit un socle de références largement commun à tous les Espagnols ou Portugais s'avérant, pour l'essentiel, indépendant du monde de l'école. En cela, la télévision peut se rapprocher d'une tradition orale vivante, celle qui se transmet principalement «de bouche à oreilles » comme les chansons du Festival de l'Eurovision ou du Festival da Canção Portuguesa. En somme, il n'y a rien de plus national que la culture orale télévisuelle. Le petit écran évoque d'abord une expérience de perception individuelle. A priori donc, la fréquentation individuelle de la télévision réduit le champ d'une culture commune. Pourtant, bien des références sont largement partagées, "des choses que tout le monde a vues » ou «entendues » par « ouï-dire » que Cuéntame et Conta-me savent parfaitement exploiter. Il n'est pas exagéré de dire que, dans ces séries, le recours aux images d'archives sert à la création « d'effets d'enfance ". La télévision constitue un réservoir d'allusions, de citations, de genre à imiter ou à parodier dans lequel Carlitos et ses camarades vont abondamment s'abreuver pour élaborer leurs jeux.

Le recours aux images d'archive a également été élaboré en amont comme pouvant fournir un cachet d'authenticité et donner l'illusion que le produit final est un véritable document historique. Elles sont les signes des effets de réel, des éléments paratextuels qui viennent réifier le cadre de la fiction tout en cœxistant avec elle. Elles peuvent, de la sorte, être appréhendées tantôt comme une marque de réalisme tantôt comme la documentation d'un contexte historique précis. Cependant, pareille instrumentalisation de l'archive ne va pas sans soulever des questions d'ordre narratologique ou historique puisque les images mobilisées sont simultanément des "documents" au sens foucaldien ${ }^{66}$ et des éléments du récit. Dès lors, une telle confusion est propice à l'identification des téléspectateurs. Le chercheur se penchant sur la proximité existant entre nos deux séries ne peut s'empêcher de se demander ce que le choix d'inclure telle archive plutôt qu'une autre dans la trame narrative révèle. Ne contribue-t-il pas plutôt à la promotion de certains faits historiques et à l'oubli d'autres?

Tout comme un individu, la télévision est pourvue d'une "mémoire », elle dispose de la capacité de conserver le temps écoulé. Dans cette optique, les archives comme la mémoire humaine conservent la trace de chaque événement vécu par l'homme si celuici a été perçu par la conscience ou plutôt filmé. On peut estimer que les archives audiovisuelles représenteraient autant de traces conservées du passé, des souvenirs gravés sur pellicule de ce que la télévision a pu diffuser depuis son apparition. Par analogie, les archives de la RTVE ou de la RPT peuvent donc être considérées comme une "mémoire ». Nos séries en tant qu'elles recourent à des "archives-souvenirs " peuvent ipso facto être considérées comme des mémoires spontanées. Toutefois, la mémoire s'avère assez capricieuse en ce sens qu'elle "grave » ou enregistre tous les événements à mesure qu'ils passent, en laissant «à chaque fait, à chaque geste sa place et sa date ", mais " elle met autant de caprice à reproduire que de fidélité à conserver ${ }^{67}$ ». Dans les deux versions de la série, par le truchement de l'objet télévision, « le passé se succède à lui-même dans un présent qui le répète simplement sous une autre forme ${ }^{68}$ ». Dans tous les épisodes, les personnages et leurs actions se nourrissent de ce qu'ils ont vu auparavant, des évènements qu'ils leurs ont été rapportés durant les bulletins d'informations TV. En quelque sorte, les personnages partagent cette "mémoirearchives-souvenirs » avec les téléspectateurs qui sont par ce biais invités à aller puiser dans leurs propres souvenirs télévisuels pour favoriser leur immersion active dans la 
fiction. En agissant de la sorte, le passé proche de l'Espagne et du Portugal peut être lu à travers la médiation de Cuéntame cómo pasó et Conta-me como foi, "sur un mode "participatif" " - «ça s'est passé exactement comme dans un épisode de...»-, «qui permet aux spectateurs de faire advenir la fiction dans le réel et d'y participer ${ }^{69} »$. Les archives disposent, en effet, de cette possibilité extraordinaire de «conserver le passé dans un présent qui s'en enrichit» disait $\mathrm{H}$. Bergson. Si l'on continue à filer la métaphore, on serait ainsi tenté de dire que les showrunners ont, avant tout, cherché à répondre à des objectifs inscrits dans le présent (esthétique, économiques avec le public ciblé, les audiences ou parts de marchés, la rentabilité entraînant merchandising et autres produits dérivés). Ce présent reste le temps des téléspectateurs. Par conséquent, s'ils font appel aux images passées, aux "souvenirs-archives", s'ils les sélectionnent c'est en vue de préparer la meilleure réponse aux sollicitations extérieures des téléspectateurs. Ce travail de sélection des documents d'archives équivaut à une sélection des faits pouvant décemment être comparée à l'oubli.

\section{Les discours véhiculés sur les transitions démocratiques ibériques ou l'intertextualité mémorielle}

\section{A - Sur l'utilisation problématique des archives}

Cuéntame et Conta-me réactivent le passé à la lumière du présent et, par-là même réécrivent l'histoire. La réactivation de la «mémoire historique ${ }^{70}$ " passe par le truchement de la télévision publique. Les archives audiovisuelles choisies pour éclairer les téléspectateurs du $\mathrm{xxI}^{\mathrm{e}}$ siècle permettent d'observer et de mesurer l'importance attribuée à certains événements historiques. Le silence sur d'autres faits résulte bien de ce que les showrunners sont, pour reprendre Marc Ferro, "attentifs à servir, consciemment ou non, une cause, que ce soit leur Église, leur patrie, leur parti, voire leurs simples convictions ${ }^{71}$ ». Le recours à la « mémoire-archives » de la télévision est susceptible de servir une cause, de souligner des dysfonctionnement du temps présent voire, de conforter des prises de positions.

Pensant sans doute refléter une vision largement partagée par le public qu'elles visent, nos deux séries donnent à voir le rapport à la conscience qu'une communauté nationale peut entretenir avec son propre passé. La reconnaissance ou l'oubli peuvent fonder la respectabilité d'une communauté nationale à un moment précis de son histoire. Dans ces circonstances, il est important de rappeler que dans nos contrées occidentales, la télévision s'apparente souvent à un média de "reconstruction nationale ${ }^{72}$ " depuis la Seconde Guerre mondiale. En ce sens, elle est un vecteur essentiel du « récit national ${ }^{73}$ ». Par conséquent, en faisant du poste de télévision l'un des personnages principaux du récit, les deux versions ibériques de Cuéntame contribuent à l'élaboration d'une mémoire collective consensuelle sur les dernières années de la dictature et de la transition vers la démocratie. Dans ces deux productions, on ne saurait parler d'une « histoire populaire » à la manière de Howard Zinn ou Gérard Noiriel comme on peut le croire en lisant les informations disponibles sur les différents portails web spécialement créés pour leur lancement ${ }^{74}$. Les showrunners n'ont pas souhaité mettre l'accent sur la place et le rôle du peuple dans tous les grands évènements, les luttes sociales et syndicales qui ont scandé l'histoire de l'Espagne et du Portugal ces quarante 
dernières années. La recherche de la lisibilité n'a pas signifié qu'il ait été question de faire de l'histoire savante sous forme ludique, mais bien de présenter un «roman national » fondé sur une vision quelque peu anachronique du passé afin de permettre l'identification du téléspectateur d'aujourd'hui.

Si l'année 1968 marque la «chute » de sa chaise de Salazar et l'accession de Marcelo Caetano à la présidence du Conseil au Portugal, il n'en reste pas moins que l'année 1968, signalée par les Carlitos comme "une année révolutionnaire ", n'a eu qu'un modeste écho dans l'ensemble de la péninsule ibérique. En 1968, très peu de familles de la classe moyenne possédaient les moyens de s'offrir une télévision ou un appartement équipé. $\mathrm{Au}$ prix d'un décalage temporel qui, quarante ans après, ne semble gêner que les historiens, les showrunners font entrer les Espagnols et les Portugais dans l'image que les Européens du bloc occidental se font des Trente glorieuses. Le niveau de vie des ibériques était alors similaire aux pays sous-développés, qu'on appelait "TiersMonde ». Ajoutons à cela à la nature autoritaire des régimes avec leurs «mesures de sécurité préventives ", polices politiques, systèmes de censure, etc., qui entraînent de facto un éloignement de toute préoccupation politique. Le chômage y a toujours existé mais la censure des régimes de Franco et Salazar contribuait à masquer cette réalité-là et installait un climat composé de résignation et d'attentisme. À l'aune de ces faits, on voit bien que les réalisateurs espagnols et portugais rusent avec les années écoulées pour se mettre au diapason des télévisions ouest-européennes qui, presque unanimement, reviennent sur ce qui se passait avant le choc pétrolier de 1971.

La reconstitution du passé qui est proposée par Cuéntame et Conta-me, en plus d'être fondée sur des anachronismes, constitue une vision partielle et partiale du passé. Ces deux séries développent en effet un discours nostalgique et consensuel alors que l'histoire contemporaine, comme discipline scientifique, se revendique plutôt comme histoire des ruptures et des traumatismes collectifs. L'historien du temps présent travaille certes avec la mémoire - le témoignage - comme source, parmi d'autres ou à défaut d'autres. Toutefois, il le soumet à l'examen critique, l'histoire qu'on dit «immédiate » requérant les mêmes méthodes de travail que l'histoire des autres périodes. Les souvenirs rapportés par nos deux Carlos interpellent donc le chercheur à plusieurs égards. D'abord, les propos tenus sur le passé valent pour eux-mêmes, en dehors de tout cadre référentiel. Le témoignage de Carlos n'est jamais subordonné à la logique du traitement historique que l'on peut retrouver dans un documentaire par exemple. Le passé n'est que "son" passé et ne peut objectivement prétendre à la généralisation car il n'a de sens que pour celui qui l'exprime. De plus, il n'est jamais confronté à d'autres points de vue, d'autres souvenirs que les siens. En se focalisant sur le passé particulier d'une famille, qu'il s'agisse des Alcántara ou des Lopes, le narrateur ne peut pas prétendre à l'objectivité contrairement à ce qu'affirme le portail de Cuéntame cómo pasó. Carlos nous raconte la "petite histoire » de sa famille en veillant tout de même à apporter quelques éléments de contextualisation, qu'il aura préalablement sélectionnés dans la culture populaire télévisuelle pour faciliter la compréhension de son propos. Les extraits qu'il a choisis mettent le plus souvent l'accent sur les actualités sociale (les objets de consommation, type lave-linge, les premières greffes d'organes, les hippies, etc.) et culturelle (le remariage de Jackie Kennedy et autres " actualités people » en général, etc.) pour renforcer l'idée du bienvivre de cette époque. De tels choix ne font que contribuer à la propagation de la saudade. Les images d'archive nous tirent vers l'arrière, on y évoque des époques révolues en évitant soigneusement l'Histoire, les réalisateurs évitant toute tentative 
pour mettre en perspective les évènements et comprendre comment ils ont pu s'enchaîner. Le propos final se voit ainsi dépourvu de toute visée critique et constitue presque une forme de renoncement au processus de «récupération de la mémoire historique » et de réparation morale.

La matière audiovisuelle est exploitée sans que soient prises en considération les intentions à l'œuvre dans les archives, leur part de subjectivité ou leur teneur idéologique. Les images de propagande d'hier sont remontées comme si elles étaient «transparentes » c'est-à-dire porteuses du véritable sens de l'histoire. Les réalisateurs utilisent le document ancien comme s'il pouvait instaurer un contact direct, sans filtres, entre le téléspectateur et la réalité historique du passé. On pense ici à l'utilisation qui est faite dans l'épisode 15 de Conta-me des "conversas em familia de Marcelo Caetano", un programme de "commentaires politiques " d'une vingtaine de minutes, diffusé en première partie de soirée à partir du 8 janvier 1969 jusqu'au 28 mars 1974, pendant lequel le président du Conseil expliquait à la nation le sens de ses réformes et pourquoi il était nécessaire d'envoyer des contingents de conscrits dans l'outremer. Ces documents sont présentés aux téléspectateurs tels quels. Les archives vidées de leur substance idéologique, utilisées pour animer le cadre, abandonnant aux seuls personnages le soin de formuler des commentaires. Or, le mantra " aqui no se habla de politica » ou « aqui não se fala de politica » ${ }^{75}$, inlassablement répété par l'ensemble des adultes et censé rendre perceptible la surveillance généralisée, la suspicion permanente, la crainte d'être entendu et dénoncé par les voisins, la peur d'avoir affaire à la police, fait que la valeur du document est réduite à une fonction strictement illustrative.

Comme le souligne l'historien Marc Ferro, «tous les documents doivent être analysés comme des documents de propagande [...] Les images d'archives ne sont pas mensongères au moins sur un point: ce que l'on a voulu dire aux gens ${ }^{76} »$. Les showrunners n'interprètent pas les archives qu'ils choisissent de montrer à l'écran omettant sciemment qu'elles sont issues des fonds de la RTVE et de la RTP, chaînes créées à l'initiative des pouvoirs publics dictatoriaux comme des attributs culturels de leur souveraineté étatique. Ils espéraient par-là contribuer à leur normalisation, à obtenir un minimum d'adhésion de la part de leurs peuples. Pour éclairer notre propos, il n'est pas inutile de rappeler que la RTVE ou la RTP, qui produisent les deux séries, ont, de plus, été les seules chaînes de télévision nationales jusqu'au début des années 1990. Les images d'enregistrement qui figurent dans leurs fonds d'archives sont des interprétations $\mathrm{du}$ réel et ont, pour cela, un rôle éminemment politique. Elles constituent des interprétations de la réalité historique sous forme de divertissement des masses populaires au service de la vision du monde et des intérêts salazaristes et franquistes. Les showrunners sombrent dans l'illusion de transparence de l'archive dénoncée par Liora Israël pour qui "l'archive ne "dit" rien (...), hors d'une problématique et d'une réflexion donnée ${ }^{77} »$.

50 La nostalgie suscitée peut ainsi être assimilée à une entreprise de banalisation du régime, banalisation d'autant plus grande qu'on fait ressortir que le véritable ennemi est le libéralisme économique. En fonction de cette conjoncture politique et économique nouvelle, on peut ainsi refouler Salazar, Caetano ou Franco grâce aux politiques néolibérales et autres plans d'austérité subis à répétition depuis les années 1980. Ces hommes forts sont mythifiés et désormais présentés comme des hommes âgés et malades qu'on importune inutilement. Les délateurs, les agents d'information des 
polices politiques, les arrestations arbitraires, les tribunaux spéciaux, les interrogatoires, les tortures s'effacent devant l'ordre et la paix fantasmés comme si le point de vue porté sur cette période était resté celui d'un enfant de 8 ans.

51 Le coût humain et social des deux dictatures est ici largement minoré et le traitement qui en est fait se veut "grand public» et verse inlassablement dans le localisme, l'anecdotique, le recentrage sur l'individu. Les causes collectives disparaissent au profit des seuls liens familiaux ou d'amitié signifiant expressément que la sphère privée prime sur la volonté de se situer par rapport au monde et par rapport au temps. Dès lors, s'il est bien question du passé dans Cuéntame et Conta-me, il n'est nullement question d'Histoire. C'est un passé restreint au cercle de la famille et des voisins, un passé limité à la mémoire en somme. La référence commune est le "nous ", "nos " années, ce que "nous » étions. Le «nous » du récit est englobant, il inclue l'entourage proche, mais demeure temporellement limité. Le passé ne remonte pas plus loin que ce que le "nous» a été en mesure de rapporter, ce que les témoins oculaires ont pu témoigner de ce qui leur était arrivé. S'il faut se garder d'une analyse marxiste primaire, force est de constater que le «nous " récurent dans la narration cherche d'abord à renvoyer au «nous, le peuple espagnol » ou «nous, le peuple portugais », en présupposant que les individus constituant ces peuples forment une grande et belle famille, et que les autres, ceux qui ne se reconnaissent pas dans ces récits, sont rejetés en dehors de «l'hispanité » ou de la « portugalité ».

Alors que les séries Cuéntame et Conta-me mettent en scène des personnages essentiellement fictifs, le recours massif aux images d'archives leur permet donc de se réclamer du récit historique qui doit, quant à lui, par définition, être le plus proche possible des évènements du passé, car dans l'optique des showrunners les personnages principaux ne sont pas fictifs, ils sont autant d'allégories d'individus ordinaires qui parcourent la grande histoire sans laisser de traces, et le cadre dans lequel ils se meuvent se veut véridique, les archives exhibées sont là pour en attester. Elles donnent ainsi l'illusion que le passé reconstitué est en fait une reconstitution fidèle à l'original qu'il singe, comme si, elles obligeaient les réalisateurs à adopter les méthodes de travail des historiens car, disait Georges Duby, « l'historien n'est pas libre. Il lui faut transcrire fidèlement ce qu'il extrait des documents, de toutes les traces du passé qu'il repère et qu'il lui est strictement interdit de manipuler ${ }^{78}$ ».

53 Le récit de Carlos est pareil au récit fondateur du témoin se voyant alors corroboré par le récit des showrunners ou "auteurs-historiens» qui, en recourant aux archives, s'interrogent et vérifient constamment la conformité de ce qu'ils racontent avec la réalité historique. La confusion entre le réel et les documents d'archives donne, en fait, à voir une volonté de réactiver l'impact émotionnel des événements eux-mêmes plutôt que la chaîne de causalité qui a conduit audit événement. En faisant appel à la mémoire des téléspectateurs plus âgés qui ont sans doute vu les mêmes émissions que les familles Alcántara et Lopes, on cherche non seulement à rendre proche ce qui est lointain en le conformant aux standards du flux télévisuel mais également on sacrifie au " présentisme ", c'est-à-dire cette propension très actuelle à rapprocher l'hier de l'aujourd'hui, dénoncés par l'historien François Hartog ${ }^{79}$. Dès lors, ce qui importe aux yeux des producteurs, c'est que le public du xxI ${ }^{e}$ siècle puisse reconnaître un lieu, une situation, un objet à travers une image qui lui est familière. L'efficacité narrative doit primer sur l'historicité. Un tel traitement de la réalité historique montre que pour être compris par le plus grand nombre, le passé proche doit être ressenti émotionnellement, 
par identification, l'impression l'emportant sur la connaissance abstraite et raisonnée. Nous pouvons ainsi reprendre les propos de Laurent Véray qui écrivait dans La Grande Guerre au cinéma que les archives sont devenues:

« des marchandises comme les autres, qui doivent être consommées. L'exigence de facilité, de formatage des programmes destinés à un large public, pousse de plus en plus à "actualiser" le passé, à lui donner un autre statut médiatique, (...) en conformant les images qui subsistent aux modalités de la perception actuelle ${ }^{80} »$.

\section{B - Deux séries qui confinent au « roman national »} travail titanesque de restitution de la «mémoire historique " entendue comme "mémoire collective ", des séries qui se veulent pédagogiques et accessibles au grand public. La «mémoire collective » qualifie une approche du passé qui n'est définie que par la distinction qu'on opère traditionnellement entre " histoire » et "mémoire " ${ }^{81}$, connaissance et imagination, objectivité et subjectivité, raison et émotion. La notion de mémoire, dans sa dimension " collective ", renvoie, quant à elle, aux représentations du passé dont les individus d'une société donnée, liés par une expérience commune, sont porteurs. Elle se forge à travers l'enseignement de l'histoire à l'école et les musées, les commémorations d'évènements considérés comme fondateurs, le patrimoine et les usages politiques du passé qu'autorisent ou non le procès, l'amnistie ou les lois dites « mémorielles ${ }^{82}$ ». Elle renvoie également aux mises en récit élaborées par le cinéma, la littérature, la télévision, "c'est-à-dire aux divers registres, didactique, politique, juridique et esthétique de la gestion visible du passé dans une société ${ }^{83}$ ». La fiction occupe une place privilégiée dans la mesure où les productions culturelles ont la capacité d'atteindre un large public et suscite l'intérêt des jeunes générations. Toutefois, la vraisemblance du cadre spatio-temporel et de la mise en scène des personnages, imaginaires ou référentiels, est davantage une arme pour manifester une opposition à la situation politique et sociale qui a présidé à l'élaboration des séries. Bien plus, nos deux séries participent non à l'écriture de l'histoire mais à celle d'un « roman national ».

Une série télévisée historique, en suivant les exemples de Cuéntame et Conta-me, a pour objet la représentation fictionnelle du passé effectif. Cependant, loin de suppléer à l'Histoire lorsque celle-ci échoue à dire ce que fut le passé des "petites gens", des individus ordinaires, Cuéntame et Conta-me ne visent que la distraction, le soubassement politique étant traité trop superficiellement. On le sait, la série TV, comme le roman, si elle a des besoins de vraisemblance n'a pas d'absolu besoin de vérité. Ainsi, les auteurs de Cuéntame ou Conta-me ont construit leur fiction télévisée comme une espèce de « roman familial» qui désigne chez Freud les fantaisies enfantines visant à cacher certains faits en imaginant un vécu plus merveilleux qu'il n'est, l'enfant s'imaginant par exemple avoir été kidnappé ou adopté, et s'invente alors des parents plus aimants. Dans nos séries, les petits "Carlitos " s'imaginent tour à tour être «le fugitif», le nouveau Lawrence d'Arabie, un joueur de foot, les reconquérants de Gibraltar ou d'Olivença, etc...

Faire de la figure enfantine le protagoniste de l'intrigue offre la possibilité aux auteurs de se dédouaner d'une mise en perspective critique des faits historiques, d'en occulter certains, ou de minimiser la portée de ceux qui paraissent incontournables en toute bonne conscience, sans courir le risque d'être taxé de révisionnisme, puisque un enfant 
n'est pas censé comprendre les tenants et aboutissants du monde des grandes personnes. Il est d'ailleurs constamment rappelé au téléspectateur que le récit est raconté du point de vue d'un enfant, le narrateur utilisant fréquemment dans ses commentaires l'expression "à cette époque, je ne comprenais pas» ou "je ne savais pas ». Mais une fois adulte, il ne cherche pas non plus à proposer une analyse des faits $a$ posteriori. L'adulte qui raconte son enfance reste pris dans le discours de ses parents, c'est-à-dire reste un enfant qui ne disposerait que du discours parental pour rendre le monde autour de lui intelligible. Les propos des Carlos se construisent à l'aide des signifiants en souffrance du côté des parents et grands-parents sans jamais les remettre en perspective. Par ce procédé, ces deux séries se veulent ainsi apolitiques, à l'image de leurs héros. L'absence ou la minimisation de culture politique favorise un type particulier de lecture : celui allant dans le sens du «discours officiel» ou plutôt du « mythe officiel » élaboré autour des dynamiques de transition démocratique.

Pour autant, nos deux séries tiennent un discours cohérent, intelligible qui a su transformer la naïveté, la " pureté » du petit Carlos en sagesse populaire. Le narrateur raconte ses souvenirs depuis le temps présent, un temps "dégénéré » où toutes les valeurs se sont perdues puisqu'on "abandonne les personnes âgées dans des institutions ", nous dit-il dès le premier épisode. Cuéntame et Conta-me sont des voyages émotionnels vers " le bon vieux temps ", un passé où, malgré la répression politique de la dictature, la bonté, la droiture et la simplicité rendaient les gens malgré tout heureux. Cette ode à la simplicité enfantine appelle à l'identification, à la nostalgie, à la catharsis. Juan Carlos Rueda Laffond emploie l'expression de "saccharine nostalgia " pour signifier que le but de telles productions culturelles n'est autre que perpétuer le cliché suranné des " happy sixty"; Cuénatame cómo pasó et Conta-me como foi s'abreuvant, en effet, à la source de la série américaine The Wonder Years, dont l'intrigue se déroule entre 1968 et 1974, créée par Carol Black et Neal Marlens et diffusée sur la chaîne ABC entre 1988 et $1993^{84}$. En France, elle a été diffusée par M6 à compter de 1989 sous le titre Les Années coup de coeur. Tant dans Cuéntame que dans Conta-me, la trame narrative est enrobée dans l'imaginaire collectif de l'« American dream ", c'est-à-dire le discours du «rêve américain » fondé sur une nostalgie des trente glorieuses, époque «bénie » où les simples ouvriers et employés gagnaient de bons salaires, pouvaient s'acheter des télévisions, des machines à laver, des machines à coudre, acquérir une voiture, et envoyer leurs enfants à l'université. Peu importe la conjoncture politique ou la santé des ménages, le propos s'appuie sur les valeurs entrepreneuriales telles que la persévérance et l'ambition comme pour signifier "vous aussi, si vous voulez et travaillez dur, vous pouvez devenir riche " propre au mythe américain. En effet, bien que les familles Alcántara et Lopes proviennent d'un milieu modeste, elles n'ont cessé de travailler avec acharnement, tout en consentant à faire des sacrifices. Elles se sont s'enrichies, ont acquis une maison et finalement trouvé la prospérité. Cuéntame como paso et Conta-me como foi offrent un idéal identique qui se voudrait être une représentation du rêve des petites gens travaillant dur pour faire vivre décemment leur famille. Les Alcántara et les Lopes sortent toujours vainqueurs de leurs combats quotidiens au prix d'invraisemblances flagrantes: par exemple, grâce à une grève, Carlitos obtient que le maître cesse de frapper les élèves ${ }^{85}$. En s'appuyant sur une telle rhétorique, les réalisateurs proposent une vision inoffensive et anachronique du passé dictatorial des deux pays. Ces derniers sont présentés comme devenant plus modernes et, surtout, plus européens. C'est en fait des regards conservateurs qui sont récupérés sur les dictatures ibériques donnant une représentation de l'Estado Nueve franquiste et 
de l'Estado Novo salazariste comme une époque idyllique, où trois générations pouvaient vivre en bonne entente sous le même toit malgré la promiscuité, où la solidarité régnait dans le quartier, où le curé partageait le désir de liberté de ses paroissiens. Les commentaires de Carlos Alcántara adulte, à la fin du cinquième épisode, participe de ce regard nostalgique, idéalisant cette époque passée, celle de l'enfance (et donc de l'insouciance) à jamais perdues :

Carlos Alcántara: «Mis padres crecieron bajo la dictadura en un país destruido. Conocieron el hambre y la miseria y se pasaron la vida luchando para que sus hijos tuvieran un futuro mejor. Gracias a ellos, mi generación ha sido más libre y más próspera pero no sé si más feliz ${ }^{86} »$. morale de l'histoire qu'il vient de raconter afin de nous faire regretter l'époque décrite. Certes, le regard rétrospectif peut être considéré comme la raison principale d'une telle vision du passé proche. Les leçons données par les personnages des grands-mères ou des pères de famille sur la nécessaire mémoire, la conclusion des épisodes 7 (Cuéntame) ou 9 (Conta-me) sur la perte de la peur de s'endetter et le retour des espérances (qui fait écho à la l'acceptation de contracter des crédits) sont en quelque sorte anachroniques tant elles traduisent des points de vue post-transitions. Ce recours à de tels artifices permet aux séries de jouer à fond la carte du "rétromarketing " pour séduire les téléspectateurs. La RTVE et la RTP sont parties d'un constat assez simple : à l'image de certains produits alimentaires phares de nos enfances qui, après avoir sombré dans les abysses de l'oubli pendant des décennies, reviennent aujourd'hui dans les rayons de nos supermarchés tels que le chocolat en poudre Banania ou la poudre Cérélac portugaise, la nostalgie à la télévision est devenue rentable. Cela peut en partie s'expliquer par un besoin d'enracinement qui s'exprime chez des téléspectateurs effrayés par la complexité de la mondialisation et ses enjeux. Dans les temps difficiles de la crise 
économico-financière et de l'explosion de la bulle immobilière, le «retour vers le passé » permet de se divertir au sens pascalien du terme et d'oublier les insatisfactions du présent autant que les peurs de l'avenir.

Le passé dans nos deux séries se veut donc confortable et sympathique. Derrière la saudade, la nostalgie, qu'elles véhiculent se tapie la sécurisation d'une époque où l'autarcie économique, le replis identitaire permettaient de se tenir loin du tumulte de la société marchande et de l'agitation sociale caractéristiques des démocraties occidentales. En nous permettant de retrouver un souvenir de notre enfance par le biais d'un programme TV, d'un vêtement ou d'un objet emblématique de l'époque, ces séries nous feraient nous sentir bien. Dès lors, Cuéntame et Conta-me créent une émotion et entrent plus rapidement dans le cœur des téléspectateurs. En effet, qu'il y a-t-il de plus affectif que d'évoquer des "madeleines de Proust», des souvenirs d'enfance qui auraient pu être les nôtres si nous étions nés à la même époque ? La nostalgie se trouve également renforcée par l'appel constant à l'humour et aux sentiments plus qu'à l'histoire et la politique. Il se crée alors une ambiguïté sur l'utilisation de l'enfant comme narrateur et « résistant malgré lui » et la description de son univers. Celui-ci est d'emblée présenté comme rassurant puisque le téléspectateur sait, d'une part, que l'enfant a atteint l'âge adulte sans trop de blessures, d'autre part, qu'il aime à se replonger dans les symboles de son monde de jadis. Carlos, en authentique «enfant de la télé ", s'imprègne de tout ce qu'il voit et entend et reproduit par conséquent avec maladresse tantôt l'idéologie franquiste (ou salazariste), tantôt celle des résistants, opposants à la dictature. Ainsi, les épisodes "Non plus ultra" (S01E9) et "Saetas y torrijas» (S01E20) nous le dépeignent en enfant exemplaire espérant gagner un voyage à Rome ou tentant de faire tous les sacrifices recommandés durant la Semaine Sainte (on retrouve d'ailleurs ces mêmes situations dans Conta-me como foi aux épisodes "Mini, Maxi, Ultra» (S01E7) et "A Semana da Paixão » (S2E11). À l'opposé, dans l'épisode "Une larga espera» (S01E24), Carlos projette d'enlever Doña Carmen Polo, épouse du Caudillo en visite dans son école, en l'attirant à l'aide d'un collier en fausses perles, pour réclamer la libération de son frère Toni ${ }^{90}$. On retrouve une situation analogue dans Conta-me, "A Longa Noite» (S02E15), dans lequel Carlos décide d'enlever Dona Gertrudes Tomaz, femme du président de la République, l'Amiral Americo Tomaz, en l'appâtant avec un bouquet de fleurs pour l'échanger contre la libération de son frère Toni. Cette imitation déformée des visions du monde des adultes par Carlos, ce "gloubi-boulga $a^{91}$ " ou confusion idéologique, favorise un conformisme social et ses postures conservatrices dans la narration charriant de facto un révisionnisme.

Les producteurs n'ont cessé de revendiquer à cors et à cris des expertises historiques rigoureuses et ont mis en avant le travail de leurs conseillers historiques (Laura Pousa, Helena Matos). À vrai dire, Cuéntame et Conta-me construisent des récits historiques linéaires, faits d'anecdotes et brassant de nombreux stéréotypes. Le franquisme disait de lui-même, tout comme le salazarisme, qu'il n'était pas une dictature comme les autres et, après une période d'instauration d'une sévérité qu'elle avait estimé nécessaire, elle aurait apporté la paix et la prospérité au pays. Cuéntame présente les dernières années du régime franquiste comme une "dictablanda ( " une dictature molle»), une "dictature assagie » là où Conta-me, verse dans le poncif rebattu du " Portugal, pais de brandos costumes» («Portugal, pays aux mœurs suaves») qui a abondamment servi à l'époque salazariste pour atténuer l'effet des guerres coloniales et en rejeter la responsabilité sur autrui, les «terroristes à la solde de l'étranger 
[soviétique]». Le franquisme et le salazarisme ont diffusé l'image de régimes autoritaires certes mais "paternalistes ", c'est-à-dire guidés par le souci du bien-être de leur peuple respectif. En présentant les sociétés espagnole et portugaise comme des sociétés de consommation, ces séries contribuent à propager l'idée que le général Franco ou Marcelo Caetano auraient créé une société nouvelle sur la base d'un développement économique dynamique et jeté les bases matérielles nécessaires à la construction démocratique. Les séries reconduisent le discours de propagande sur les bienfaits de l'ordre, la paix sociale et le développement économique. Elles ne s'attachent nullement à montrer les responsabilités des dirigeants ou des fonctionnaires, en particulier celle des militaires et des agents de la police politique ou de la Guarda civil, c'est-à-dire les acteurs de la répression politique et sociale. On est même tenté de dire que dans le cas de Cuéntame cómo pasó, les showrunners espagnols, ne tenant pas compte de l'historiographie, ont évacué la question des responsabilité du régime franquiste en reconduisant le «pacte de $l^{\text {'oubli }}{ }^{92}$ " instauré après la promulgation de la loi d'amnistie politique du 14 octobre 1977.

Globalement, Cuéntame et Conta-me donnent à voir un imaginaire enfantin pétri de nostalgie pour un monde perdu faisant que le monde académique tant espagnol que portugais a souligné le "révisionnisme » propre à ces séries. Le recours aux fonds d'archives montre en effet que tant Cuéntame que Conta-me ambitionnent d'être des histoires nationales au "deuxième degré ». Bien plus, elles reflètent une histoire contemporaine de l'identité nationale (espagnole ou portugaise) dès lors que dans le récit des Carlos, mémoire et identité vont de pair, la trame narrative ne laissant guère de place aux conflits et aux fractures. Le message véhiculé dans Cuéntame cómo pasó traduit la volonté de revivifier une lecture binaire et simpliste de l'histoire fondée sur l'oubli des "mémoires des vaincus» lors de la guerre civile. L'hispaniste Florence Belmonte n'a pas hésité à qualifier, comme beaucoup d'autres critiques, la série de "néo-franquiste " $^{93}$. On y trouve le souci de solder les comptes du passé, de formuler une rhétorique de l'apaisement et de la résolution des conflits entre les "vainqueurs » et les «vaincus " d'hier, une volonté de réduire les ressentiments. Cuéntame cómo pasó cherche, en ce sens, à influencer la mémoire collective espagnole pour restaurer une communauté nationale encore perçue comme déchirée en se focalisant sur les seuls souvenirs de l'expérience partagée par le narrateur mais aussi les téléspectateurs à travers la culture populaire télévisuelle. Cuéntame propose une reconstitution des dernières années de la dictature franquiste hors-sol, une histoire mythique délibérément située dans la mémoire c'est-à-dire dans l'émotion et en dehors de l'Histoire.

En reprenant l'analyse de Sophie Baby ${ }^{94}$, Cuéntame cómo pasó reconduit le «mythe de la Transition pacifique et consensuelle, menée sans heurts ni violence », un "modèle de transition en douceur » qui glorifie les élites politiques venues de la dictature - le roi Juan Carlos, Adolfo Suárez, Torcuato Fernández-Miranda ou Santiago Carrillo - qui a été asséné à l'envi depuis la mort du Caudillo en 1975 et a fini par devenir une "vérité " au mépris de la réalité historique. Pareil discours a été légitimé et abondamment relayé par les sciences sociales et la science politique en particulier depuis les travaux de Juan Linz, jusqu'à la "transitologie » des années 1990 et 2000 inaugurée par Philippe Schmitter. Ce courant est, à l'heure actuelle, fortement critiqué par l'historiographie. La série espagnole relaye donc une "Transition modèle " fondée sur un compromis ayant rendu possible une entente cordiale entre les différents partis et acteurs politiques portant sur les modalités du nouveau régime démocratique à mettre en place 
tout en refusant de montrer les "violences politiques en transition ${ }^{95}$ ", les 3200 actes violents (attentats, menaces, émeutes, protestations, troubles dans l'espace public, etc.) perpétrés tant par l'opposition (extrême droite nostalgique du franquisme, militaires putschistes, gauche révolutionnaire, notamment anarchiste, mouvements nationalistes radicaux comme les indépendantistes canariens du MPAIAC ou les terroristes basques de l'ETA) que les organes répressifs de l'État (police, armée, garde civile). Cette violence occultée a fait 714 morts pour des raisons politiques et a profondément marquée la période entre 1975 et 1982. Dans la série, nulle mention n'est faite de la " guerre sale » conduite par l'État démocratique espagnol dans la « lutte contre le terrorisme ». On n'y trouve pas non plus de trace de l'épisode des Groupes antiterroristes de libération (GAL) que Sophie Baby qualifie de «terrorisme d'État (...) incontrôlé et mercenaire » soulignant ainsi la continuité entre la période franquiste et la transition démocratique. Même si la série évoque certains attentats, notamment ceux de l'ETA, dans l'ensemble selon Cuéntame, il n'y a pas eu en Espagne de violence pouvant être décemment comparée à celle des « années de plomb » en Italie car une culture du consensus aurait tout bonnement remplacé une tradition du conflit et du pronunciamento. L'image d'une Espagne en proie au chaos, traversée par des périodes de violence politique extrême comme celle de la Guerre civile (1936-1939) a, en fait, cédé la place à celle d'une Espagne réconciliée, apaisée et pacifique. Dans la série, la violence est toujours considérée soit comme un phénomène sporadique (le coup d'État manqué du lieutenant colonel Tejero du 23 février 1981 - le «23F »), soit comme émanant de groupuscules terroristes comme l'ETA qui ont refusé le processus de démocratisation. La violence politique apparait comme « une aberration périphérique qui rend encore plus éclatante la cohésion du reste de l'Espagne ${ }^{96} »$. Dans Cuéntame, la plupart des souvenirs de la violence politique de la période de la Transition « ont disparu de la mémoire collective, broyé sous le poids du mythe institutionnel ${ }^{97}{ }^{7}$. Cuéntame force ainsi le trait de la réconciliation et de la paix sociale en niant les faits de violence qui sont pourtant au cœur de l'histoire politique espagnole récente. En cela, la fiction reprend pleinement tous ses droits en s'inscrivant contre l'évolution historiographique. Il s'est ici agi de promouvoir le discours de réconciliation symbolique forgé dans les années de la Transition pour refermer une déchirure originelle, un discours qui repose sur un système de représentation bipolarisé fondé sur une mythification de la figure du Caudillo " pourvoyeur de paix » et " rassembleur de la communauté nationale » au-delà des divisions fratricides d'hier, bien plus que sur une critique explicite des idées et du comportement des vainqueurs de la Guerre civile.

La version portugaise, quant à elle, retrace les dernières années de l'Estado Novo (1968-1974), les années dites marcelistes et verse allègrement dans le «mythe de la transition démocratique manquée». Elle promeut une vision "branqueada», disculpante et optimiste de l'ultime période de la dictature. Conta-me como foi se focalise ainsi sur la "primavera marcelista" (le "printemps marceliste») et insiste particulièrement sur le lancement des grandes œuvres publiques, la construction d'immeubles d'habitation à Lisbonne et la croissance économique de la période. En effet, si le PIB a augmenté en moyenne de 7,5\% entre 1968 et 1973, ce chiffre étant à peine dépassé par la Grèce ( $8 \%$ ) et le Japon $(9,5 \%)$, soit en valeur absolue, le PIB per capita est passé de $275 \$$ en 1970 à $1217 \$$ en $1973^{98}$. Malgré un taux de croissance mirobolant, qui a réduit le sous-développement chronique du pays vis-à-vis des autres pays européens occidentaux, le Portugal restait le pays le plus pauvre de l'Europe de l'ouest. Des salaires très bas, un pouvoir d'achat très faible et surtout une mobilisation 
massive pour la guerre coloniale qui a contraint des centaines de milliers de Portugais à émigrer. On estime que près de 100000 personnes accomplissaient tous les ans le " salto » c'est-à-dire quittaient clandestinement le pays. Entre 1960 et 1974, près de $40 \%$ de la population active a fui le pays ${ }^{99}$. Initialement, le programme d'action du nouveau gouvernement avait pourtant provoqué, au sein de la population, l'espoir de voir enclenchée une véritable réforme institutionnelle qui conduirait le régime vers plus de démocratie et la fin des guerres coloniales. Cependant, tout ceci est resté, dans les faits, lettre morte.

Dans la série, le régime est présenté comme un seul bloc, comme une entité pérenne dans laquelle les dissensions semblent inexistantes. Les auteurs ont délibérément choisi d'axer leurs propos sur la modernisation économique et sociale en insistant également sur la timide libéralisation politique entreprise par le nouveau président du Conseil. Mais, ils délaissent les faits qui ne cadrent pas avec l'idée que l'on se fait traditionnellement des Trente glorieuses. Le parti unique, l'União Nacional rebaptisé Acção Nacional Popular en 1970, disposait d'une aile « libérale » ouvertement réformiste à laquelle appartenaient d'importantes figures comme Francisco Sá Carneiro, Francisco Pinto Balsemão, Miller Guerra, Magalhães Mota, Pinto Leite ou Mota Amaral qui ont tous siégé comme députés. La poursuite des opérations militaires dans l'outremer (Mozambique, Guinée-Bissau, Angola, Cap-Vert, São-Tomé e Principe) a fait que l'opposition entre les « réformateurs » de l'aile libérale et les « intégristes » est devenue béante et contribua à la chute de l'Estado Novo. Le slogan porté par Caetano "l'évolution dans la continuité » restait, dans les faits, synonyme du « vivre habituellement » cher à Salazar. Selon les mots de l'historien Sottomayor Cardia, le marcelisme n'était rien d'autre qu' " un salazarisme déguisé qui est rapidement apparu comme un salazarisme désorienté100 ${ }^{\prime}$. M. Caetano a supposé qu'il était possible d'orchestrer une ouverture politique et sociale tout en maintenant les principes fondamentaux paternalistes, autoritaires et corporatistes du régime salazariste et ce, même s'il a autorisé le retour au pays de l'évêque de Porto, António Ferreira Gomes, et de Mário Soares.

Si la version portugaise a été beaucoup moins critiquée par les historiens, c'est seulement parce qu'elle n'a pas connu le succès de son homologue espagnole. Elle n'en propose pas moins une vision irréelle de l'époque marceliste (1968-1974) et des guerres coloniales en particulier. Traitant d'une période faisant relativement consensus tant parmi le grand public que le monde académique, la série s'est bien gardée d'évoquer des évènements clivants comme les crimes de la guerre coloniale, les luttes intestines au sein de l'appareil d'État, l'après 25 avril et la décolonisation, les dérives du « PREC » ou " processus révolutionnaire en cours » qui dura près de deux ans et demie ainsi que le sort des "retornados" - « rapatriés » des anciennes colonies. Les dernières années d'existence de l'Estado Novo ont été recouvertes d'un vernis chatoyant comme si la période avait été un chrononyme ${ }^{101}$ heureux ponctué par des progrès sociaux, économiques, technologiques et politiques alors qu'elle reste fondamentalement associée à l'oppression de la police politique, la tristement célèbre Pide/Dgs, à la "police de choc», à l'injustice, au sous-développement chronique du «vivre habituellement » et, surtout, aux treize années de la Guerre coloniale. La longueur de ce conflit explique pourquoi les auteurs de la série n'ont pu éviter à Toni, le fils aîné des Lopes, d'accomplir son service militaire. Toni, le grand frère du narrateur, jeune étudiant en droit s'est peu à peu conscientisé après son entrée à l'université. Il en est venu à participer à des « réunions politiques » ou des « veillées pour la paix ${ }^{102}$ ». Après avoir fait ses classes, il est donc envoyé sur le front mozambicain pour «salvarguardar o 
que é nosso ». La série ne remet jamais en question le pourquoi de la guerre coloniale. Seulement, dans la mesure où celle-ci était susceptible d'affecter personnellement toutes les familles ayant des fils en âge de porter les armes, traiter de cette réalité était inévitable. C'est d'ailleurs la prise en compte des guerres coloniales qui éloigne la version lusitanienne de l'originale espagnole en lui conférant une timide coloration critique. Toni reçoit donc son ordre de mobilisation quelques semaines seulement avant la Révolution des CEillets $^{103}$. Les auteurs n'ont pas voulu se risquer à tenter d'élaborer une représentation sophistiquée du phénomène guerrier et se sont contentés de filmer le banal et la quotidienneté de la vie au front. La violence de la bataille est mise à distance, réduite à des bruits hors-champ, évitant ainsi toute possibilité de spectacularisation. Toni n'est montré qu'en train d'écrire des lettres à sa famille ou lisant le courrier qu'il reçoit. Ce faisant, les auteurs tombent dans l'écueil de la déréalisation. Les défaites et les errements sont absents, les atrocités commises par les forces armées ne sont pas évoquées... Les mouvements de libération africains sont combattus par une armée portugaise composée de 150000 à 192000 hommes, fortement équipée en moyens de destruction modernes: jets-bombardiers, hélicoptères, navires de guerre, chars, mortiers, bombes à fragmentation, au napalm, au phosphore blanc et défoliants (herbicides déversés sur les zones « libérées » afin de saccager les récoltes et de briser la résistance des populations en les affamant). À aucun moment dans la série, notamment lorsque Toni se retrouvera sur le front mozambicain, il n'est fait allusion à ces pratiques et équipements armés. Il était nécessaire de préserver l'aspect bucolique jusqu'au dernier épisode car, il s'agit d'une comédie familiale, pas d'un drame.

La série refuse également de montrer que la guerre coloniale a été le prétexte de l'aliénation des libertés publiques des citoyens portugais. Elle a donné l'occasion au régime de maintenir le parti unique, renforcer la censure dit lapis azul ${ }^{104}$, la police politique rebaptisée "Direcção-Geral de Segurança» et la répression accrue envers les opposants. Le gouvernement a entrepris une violente répression contre les mouvements sociaux et partis politiques tels que la CDE - la Commission Démocratique Électorale (vaste éventail allant de la gauche communiste jusqu'aux catholiques sociaux) - ou la CEUD - Commission Électorale de l'Unité Démocratique (qui incluait certains membres fondateurs du futur PSP). La répression policière, le recours aux «tribunaux spéciaux» tenus par la PIDE provoquent des désertions massives et l'émigration massive des hommes en âge de partir pour le front. La série n'évoque pas non plus l'isolement dont souffre le Portugal estadonoviste sur la scène internationale du fait de son acharnement à «lutter contre le terrorisme » dans les "provinces d'outremer » comme les désigne désormais la propagande d'État. Les mouvements d'opposition à la guerre sont très brièvement considérés lors de l'épisode "Ano novo " lorsque Toni se rend à une veillée dans l'Église du quartier organisée par le père Victor pour la paix, mais ni les désertions ni les fugues des appelés vers l'étranger ne font parties du scénario. D’ailleurs, de manière plus générale, la contestation étudiante disparaît de la série après que le personnage de Lena, la première petite amie de Toni ayant perdu son bébé, ait quitté le Portugal pour aller vivre aux États-Unis. Si la contestation des secteurs catholiques progressistes est bien mentionnée à travers la figure du prêtre Victor, elle disparaît également lorsqu'il entame une relation amoureuse avec Isabel Lopes. Enfin, les protestations de l'aile libérale du régime n'existent pas dans Conta-me, les démissions de Costa Gomes ou du général Spinola ${ }^{105}$ n'attirant pas l'attention des scénaristes. Seul le livre de Spinola, "le général au 
monocle ", O Portugal e o futuro est évoqué puisqu'il s'agit de la première grosse commande de l'imprimerie tenue par António Lopes et ses associés. Cependant, nous pensons que cet ouvrage a été mentionné non pas pour son contenu, qui pouvait sembler subversif en 1974, mais parce qu'il a été un véritable succès de librairie, signifiant de la sorte les retombées financières juteuses pour l'entreprise. Là-encore, aucun jugement politique, il s'est simplement agi de souligner la success story de la famille Lopes, le «nez » du patriarche pour les bonnes affaires.

$\mathrm{Au}$ cours des années 1990, les expressions «devoir de mémoire» ou «travail de mémoire " ont envahi l'espace public et la vie politique pour signifier l'obligation morale de se souvenir à l'égard des victimes de différents faits historiques traumatiques. Nous serions entrés si profondément dans "l'ère de la mémoire " pour reprendre une formule de Javier Cercas que l'historien Henry Rousso affirme que nos sociétés démocratiques modernes sont «malades » de leur passé. « La mémoire, écrit-il dans Face au passé. Essai sur la mémoire contemporaine ${ }^{106}$, constitue sans nul doute le grand mythe contemporain des sociétés démocratiques modernes, une forme de conjuration contre la répétition des catastrophes de l'histoire récente dont on commence à voir les limites ». Le « devoir de mémoire » se traduit par la publication de nombreux romans, livres de témoignages ou essais, la production de films de fiction, documentaires, reportages, l'organisation d'expositions. La fiction occupe une place privilégiée dans la mesure où les productions culturelles ont la capacité d'atteindre un large public et suscite l'intérêt des jeunes générations. De par le monde, les productions télévisées où l'histoire joue un grand rôle ne se comptent plus et, avec les séries policières, les séries historiques sont désormais le genre le plus plébiscité ${ }^{107}$. Cuéntame cómo pasó ainsi que Conta-me como foi s'inscrivent pleinement dans cette mode. Le concept original espagnol a pu être adapté dans de nombreux pays, notamment le Portugal, grâce à l'utilisation massif des fonds d'archives des chaines publiques qui les a produites. Toutefois, le recours massif aux images d'archives pour rattacher le propos à la réalité historique de l'époque est problématique car les réalisateurs ont tendance à penser qu'elle serait en elle-même porteuse du sens de l'histoire. De plus, seul le point de vue du narrateur est pris en compte faisant que le recul critique n'est pas permis.

Quelle histoire politique proposent alors Cuéntame cómo pasó et Conta-me como foi ? Nous avons insisté dans notre propos sur l'édulcoration des récits racontés qui reprennent largement à leur actif les différents mythes forgés à propos de la «dictablanda " que serait devenue le franquisme tardif et la "Transition modèle» espagnole et de la "primaveira marcelista». Dans la version portugaise de la série, la guerre coloniale s'avère totalement déréalisée. La reconstitution du passé proche dans ces deux séries obéit davantage à des injonctions mémorielles dictées par le temps présent plutôt qu'à des préoccupations historiennes. Elles montrent ainsi une mémoire reconstruite, débarrassée de tout événement collectivement marquant ou traumatisant afin de transmettre le « roman national », ciment du patriotisme. Il s'est agi de construire un propos cohérent qui soit une véritable épopée où tout s'enchaîne harmonieusement : l'arrivée de la télévision, la prospérité économique, l'avènement de la démocratie... au détriment des faits historiques. De plus, malgré le soin apporté aux décors, les auteurs ne parviennent pas à éviter certains anachronismes et font entrer de plain-pied les sociétés espagnole et portugaise de l'époque dans les Trente Glorieuses, alors que l'archaïsme des systèmes économiques de la péninsule ibérique avait fait que ces populations étaient les plus pauvres de l'Europe de l'ouest. 


\section{NOTES}

1. Dorénavant noté «Cuéntame »

2. Maria Robert, « 'Cuéntame' hace historia », El Pais, 3 mars 2016

3. Les chiffres peuvent être consultés en suivant ces liens : https://www.formulatv.com/series/ cuentame-como-paso/audiencias/ ; $\quad$ https://www.elperiodico.com/es/yotele/20180920/laeterna-pregunta-se-acerca-el-fin-de-cuentame-asi-ha-evolucionado-su-audiencia-7044420

4. La première saison compte 33 épisodes alors que la $19^{\mathrm{e}}$ en compte seulement 15 . Étant donné le succès fulgurant de la série, plusieurs saisons ont été tournées chaque année jusqu'en 2018. Fin décembre 2018, la production a confirmé le tournage d'une $20^{\mathrm{e}}$ saison. L'intégralité des épisodes est disponible ici : http://www.rtve.es/alacarta/videos/cuentame-como-paso/

5. Martin Winckler, Petit éloge des séries télé, Paris, Gallimard, collection «Folio 2€», 2012. On consultera également avec profit cet entretien que l'auteur a accordé au journal Libération disponible ici: http://feuilletons.blogs.liberation.fr/2012/09/21/entretien-avec-un-seriephilemartin-winckler/

6. Jean Bianchi, «Dallas, les feuilletons et la télévision populaire », Réseaux, vol. 3, n¹2, 1985. Dallas et les séries télévisées, p. 19-28.

7. Marine Legagneur, «Importations, transpositions, adaptation dans le feuilleton quotidien Plus belle la vie », TV/Series [En ligne], 2 | 2012. URL : http://journals.openedition.org/tvseries/1467

8. Dorénavant «Conta-me»

9. Les audiences de Conta-me como foi sont restées modestes durant la diffusion des cinq saisons oscillant entre 7 et 10,4\% des parts de marché. On compte environs 664100 téléspectateurs fidèles pour chaque épisode. Pour plus de détails, on consultera les données disponibles ici : https://www.cmjornal.pt/tv-media/detalhe/audiencias-nao-abalam-series

10. L'adaptation portugaise de la série a reçu plusieurs prix parmi lesquels le prix du « Meilleur programme » de l'année 2007 décerné par l'Associação de Telespectadores (l'équivalent des Emmy), le prix des Auteurs SPA en 2009 ou le prix Lumen de «la meilleure série de fiction de tous les temps » en 2014.

11. En février 2019, la production a annoncé la mise en chantier d'une $6^{\mathrm{e}}$ saison couvrant la période post-révolutionnaire jusqu'à l'entrée du Portugal dans la Communauté Économique Européenne (1974-1986). Voir les liens suivants : http://quinto-canal.com/televisao/conta-mecomo-foi-esta-de-regresso-com-nova-temporada ; $\quad$ https://www.publico.pt/2019/02/12/ culturaipsilon/noticia/contame-regressa-rtp-passa-ditadura-anos-80-1861634

12. Adélaïde Pestano y Vinas, "Le récit-estampes ", Mélanges de la Casa de Velázquez [En ligne], 39-2 | 2009. URL : <http://journals.openedition.org/mcv/2994>

13. Terme littéraire intraduisible qui désigne les récits décrivant le quotidien et les mœurs d'une époque

14. Adélaïde Pestano y Vinas, « Le récit-estampes », op. cit.

15. Mona Ozouf, « Récit des romanciers, récit des historiens », Le Débat, n¹65, 2011, p. 13.

16. Voir e.g. "Quand le petit écran explore le temps ", La revue pour l'histoire du CNRS [en ligne], 24 | 2009, URL : http://journals.openedition.org/histoire-cnrs/9165

17. Laura Pousa, «Tell the (hi)story to the nation. Two transcultural adaptations of the Spanish TV series Cuéntame cómo pasó: Raccontami and Conta-me como foi », Communication \& Society, vol. 30, $\mathrm{n}^{\circ} 2$, p. 1-14.

18. Laura Pousa a été l'une des premières scénaristes de Cuéntame. Spécialiste de l'histoire du cinéma espagnol, elle est notamment l'autrice de La memoria televisada: Cuéntame cómo pasó (Madrid, Comunicación social, 2015). 
19. Helena Matos est une historienne quelque peu controversée qui publie des chroniques dans des journaux très conservateurs comme o Observador et dont les travaux académiques portent essentiellement sur la période marceliste (1968-1974) de la dictature estdonoviste. Parmi ses ouvrages, on peut notamment citer Os Filhos do Zip Zip (Lisbonne, A Esfera dos Livros, 2013) qui est une histoire du Portugal des années 1970 à travers la culture populaire télévisuelle.

20. C. Bernard, Le passé recomposé. Le roman historique français du dix-neuvième siècle, Paris, Hachette, 1996.

21. Nicolas Offenstadt, « À bas le roman national ! », L'Histoire, collection 44, juillet-septembre 2009.

22. En France, par exemple, les programmes officiels de langue et civilisation espagnoles proposés par l'Éducation Nationale évoquent la série comme une ressource pédagogique importante pour appréhender les mœurs et la culture du pays et renvoie vers le site de El Instituto Nacional de Antropologia e Historia (INAH), qui affirme que la série « a mis à disposition pour les tous petits une série de matériaux pour qu'ils se divertissent et, tout en s'amusant, apprennent sur l'histoire universelle et de notre pays, découvrant des sites du patrimoine mondial, des zones archéologiques et des musées (...) ». Cf, http://cache.media.eduscol.education.fr/file/Espagnol/ 75/3/RA16_C4_LV_esp_declinaison_culturelle_585753.pdf

23. Charo Lacalle Zalduendo citée par Jennifer Houdiard, «Entre traumatisme originel et choc fondateur: la Guerre Civile espagnole dans la série catalane Temps de silenci (TV3, 2001-2002) », TV/Series [En ligne], 10 | 2016. URL : http://journals.openedition.org/tvseries/1858

24. Il est ici question des S01E11 (Cuéntame) et S01E12 (Conta-me) que nous analyserons plus en détail plus loin.

25. Il s'agit des S01E19 intitulés «La boz des barrio » pour Cuéntame et «A voz do Bairro » pour Contame.

26. Il s'agit des S01E18 (Cuéntame) et S02E18 (Conta-me) tous deux intitulés « Fish and chips ».

27. Il s'agit des S01E12 (Cuéntame "Un día es un día ») et S01E13 (Conta-me " Há Horas Felizes »)

28. Sylvain Trudel, «La faute épicée : quelques considérations sur le narrateur enfant dans le livre de fiction ", Québec français, $n^{\circ} 122,2001$, p. 76.

29. S01E4 (Cuénta-me, "Las invasoras ») et S01E6 (Conta-me, " As Invasões Francesas »).

30. Cette citation traduite est extraite des S01E27 (Cuéntame, "Nuevos horizontes») et S02E27 (Conta-me, « Novos horizontes »).

31. Susana Diaz, "Notas para uma lectura de Cuéntame cómo pasó » cité par Pedro Lopes, "Conta-me como foi : a (re)construção da memória histórica » in A História na ficção televisiva portuguesa, ed. Catarina Duff Burnay, Lisbonne, Universidade Catolica Editora, 2014.

32. Gérard Genette, "Vraisemblance et motivation ", Figures II, cité par Marine Legagneur, "Importations, transpositions, adaptation dans le feuilleton quotidien Plus belle la vie », op. cit.

33. Roland Barthes, «L'effet de réel », Littérature et réalité, Paris, Seuil, 1982, p. 81-90.

34. Marine Legagneur, op. cit.

35. Ibid.

36. Ibid.

37. Trésor de la langue française, Gallimard, 1990

38. Gérard Genette, Palimpsestes. La littérature au second degré, Paris, Le Seuil, 1982.

39. Séverine Barthes, Du «temps de cerveau disponible"? Rhétorique et sémiostylistique des séries télévisées dramatiques américaines de prime time diffusées entre 1990 et 2005, Sciences de l'Homme et Société. Université Paris-Sorbonne - Paris IV, 2010. Disponible sur le serveur HAL : https:// hal.archives-ouvertes.fr/tel-00574592

40. «En 1968, j'avais 8 ans. Des fois, les gens disent souvent que l'année 1968 a été le début du changement, mais il l'a certainement été pour moi. La vérité c'est qu'en 1968, je ne remarquais pas les choses importantes qui changeaient autour de moi. J'avais d'autres préoccupations, je me préoccupais, par exemple, du destin de Fugitif ». Le Fugitif est une série américaine réalisée par Roy Huggins en 1963. 
41. Marine Legagneur, "Importations, transpositions, adaptation dans le feuilleton quotidien Plus belle la vie ", op. cit.

42. Milly Buonanno, Sulla scena del rimosso. Il dramma televisivo e il senso della storia, Rome-Bari, Ipermedium Libri, 2007, p. 101

43. Milly Buonano, The Age of Television: Experience and Theories, Chicago, Intellect, 2007.

44. Il s'agit de l'épisode 8 intitulé " A Cadeira do Poder ".

45. Littéralement : « A : Ça ne sera pas sur Franco ?/T : Non papa, ça ne sera pas sur Franco/A : tu sais bien que je n'aime pas quand tu fais des blagues sur Franco/H : Tiens compte de l'avis de ton père parce qu'il a raison/T : Et si je ne la raconte qu'à la maison.../H : Mais si les voisins t'entendent ?/T : je la raconterai à voix basse/C : Allez papa! Laisse-le !/A : C'est qu'il est ... il la raconte n'importe où et il ne sait pas ce qu'il peut arriver/ $\mathrm{M}$ : Allez, Antonio, ce n'est qu'une blague/ A : Très bien, raconte ta blague mais raconte la bien/T : Ça se passe dans un bled paumé et le maire reçoit un télégramme de Madrid. Le maire, tout émotionné parce qu'il n'a pas l'habitude qu'arrive un télégramme de Madrid, le lit et dit : "Perturbation sismique détectée dans votre zone. Localisez l'épicentre. POINT"./C : Quoi ?/H : Ce garçon utilise de ces mots rares !/M : Laissez-le finir/ C : c'est que je n'y comprends rien/A : Tais-toi mon fils/T : le maire, pauvre homme qui ne comprend rien de ce qu'on lui a demandé/C : Tiens, moi non plus/M : Tais-toi/T : Bref, il a fini par dire : "Amenez-moi le chef de la Guardia Civil”. Arrive le chef de la Guardia Civil, avec son uniforme et sa petite moustache, qui dit: "Soyez tranquille, je vais trouver une solution rapidement. Nous allons envoyer un télégramme à Madrid qui dira "Perturbation sismique étouffée. L'épicentre et deux autres suspects ont été appréhendés"" ".

46. Littéralement: «quand j'étais petit, j'étais convaincu que mon père était l'homme le plus intelligent de la terre. Non seulement il avait les réponses mais les affirmait avec autorité que même mes maîtres ne pouvaient égaler. Avoir un tel père était un luxe ! ».

47. Littéralement «A.L. : J'ai déjà dit que je n'aime pas quand tu fais des blagues sur Salazar qui est un homme malade en plus/ M : mais c'est juste une blague Antonio.../A : une blague politique. Bon, tu la raconte ici mais nulle part ailleurs/ $\mathrm{T}$ : juste celle-ci. Je vais raconter. Un jour, un maire d'un petit bled paumé dans le nord reçoit un télégramme de Lisbonne. Comme il n'avait pas l'habitude de ces choses, il a mis ses lunettes et commencé à lire. "Perturbation sismique dans votre conseil. L'épicentre se trouve dans votre région"'/C : je ne comprends rien !/H : c'est ça la blague ???/T : Non, non !! Le type se met à réfléchir. Il fait venir le chef de la GNR [gendarmerie]. Il fait venir le chef de la PIDE. Il réfléchit, réfléchit, réfléchit.../C : je comprends toujours rien !/T : jusqu'à ce que le chef de la PIDE a une idée. Il a répondu au télégramme de Lisbonne:"la perturbation sismique a été suffoquée - STOP - l'épicentre et deux autres suspects ont été appréhendés - STOP" ».

48. Littéralement : «Quand j'étais petit, je croyais que mon père était l'homme le plus savant du monde. Non seulement, il avait réponse à tout mais, en plus, il les débitait avec une autorité que même le professeur Rui Braga [son instituteur] ne pouvait égaler. Avoir un tel père si savant était un luxe et une fierté "».

49. Le NO-DO est un acronyme de Noticiarios y Documentales (actualités et documentaires) que Laura Pousa définit comme « a series of cinema propaganda created in Spain during the Franco dictatorship ", cf. Laura Pousa, "Another approximation of the Spanish series Cuéntame cómo pasó:the special episodes", Matrizes, V. 11, n², maio/ag 2017, p. 1-19.

50. Littéralement : « ce qui est à nous »

51. Littéralement : « sans spectacle et sans alliances. Fièrement seuls »

52. Littéralement : «Qui a volé qui Carlitos? Sont-ce les Indiens ou alors les Portugais?»

53. Littéralement : C. A : «Comme ça, si on nous tue, on deviendra des héros de la patrie/ J : on ne peut pas être des héros vivants ?/ C : Non, parce que sinon on nous enterrent pas à Valle de Los Caidos. Maintenant, en position de surveillance!». 
54. Le traité de Tordesillas a été signé le 7 juin 1494 entre la Castille et le Portugal afin de fixer les limites de domination respective des deux puissances coloniale dans l'Atlantique. C'est en vertu de ce traité que le Brésil est revenu au Portugal et le reste de l'Amérique du Sud aux Espagnols. 55. Littéralement : «l'Espagnol s'est emparé d'Olivença ?». Pour la petite histoire, Olivenza est un petit territoire situé à la frontière hispano-portugaise qui est successivement passé sous contrôle castillan, portugais et arabe au Moyen Âge avant de devenir pleinement portugais en 1297. En 1801, pendant l'épisode dit de « la guerre des Oranges », sorte de prélude aux invasions napoléoniennes, l'Espagne a annexé «Olivença » qui est ainsi devenue «Olivenza ». Après la défaite de Napoléon, l'Espagne et le Portugal ont pourtant signé un traité par lequel l'Espagne s'engageait à restituer Olivenza au royaume du Portugal, mais la rétrocession n'a jamais eu lieu.

56. Littéralement : « $C$ : Marinho, tu dois te décider entre le garage de Lego et devenir un héros. Les héros sont déjà tous morts mais si on meurt pour la patrie on sera décorer le 10 juin et peutêtre qu'ils nous emmèneront aux Jerônimos. Allez, on se met en position de surveillance !».

57. Littéralement : « C. A : Nous allons reconquérir Gibraltar ! (...) Cervantes : Mais avant ça, vous devez venir à la manifestation de dimanche/ J.: quelle manifestation ?/Cer.: celle pour le Gibraltar espagnol! Tout le monde en sera et moi le premier !».

58. «Carlos Lopes : Nous allons reprendre Olivença aux Espagnols! Ils ne sont pas Noirs n'est-ce pas?

Camões : Non, ils sont blancs à l'extérieur mais jaune à l'intérieur ! Et le jaune, c'est la couleur de la trahison! D'Espagne ne peut venir ni bon vent, ni bon mariage ».

59. «Carlos Lopes : C'est une manifestation contre les Espagnols?

Camões : C'est tout comme!».

60. Victor Pereira, La dictature de Salazar face à l'émigration. L'État portugais et ses migrants en France (1957-1974), Paris, Presses de Sciences Po, 2012.

61. Expression forgée par André Ribaud, citée par Flore Di Sciullo, «L'archive en déplacement: une esthétique de l'écart dans les génériques de séries télévisées", Communication \& langages, n¹90, 2016, p. 85-104. URL : https://www.cairn.info/revue-communication-et-langages1-2016-4page-85.htm

62. $L '$ ' intericonicité » repose sur le principe de faire reconnaître une image connue à travers une image nouvelle. Certains parlent de « citation picturale».

63. Clément Chéroux, "Le déjà-vu du 11 septembre, essai d'intericonicité », cité par Flore Di Sciullo, «L'archive en déplacement: une esthétique de l'écart dans les génériques de séries télévisées ", op. cit.

64. Clément Chéroux, Ibid.

65. La RTVE a été créée en 1956 et la RTP, en 1957.

66. Michel Foucault élabore une théorie de l'archive dans Archéologie du savoir (Paris, PUF, 1969) qui sera ensuite reprise par Arlette Farge dans Le goût de l'archive (Paris, Seuil, 1989).

67. Henri Bergson, Matière et Mémoire, Paris, PUF, coll. « Quadrige », 1985, p. 86.

68. Ibid., p. 94

69. Antoine Faure, Emmanuel Taïeb, «Les "esthétiques narratives" : l'autre réel des séries ", Quaderni [En ligne], n88,| Automne 2015. URL : http://quaderni.revues.org/916

70. Formulation abrégée de la «Loi de reconnaissance et d'extension des droits et de rétablissement des moyens en faveur de ceux qui ont souffert de persécution ou de violence durant la Guerre civile et la Dictature » qui s'est imposée dans le débat public espagnol et qui, depuis sa promulgation en septembre 2007, ne cesse de soulever de nombreuses controverses.

71. Marc Ferro, Les documentaires et ses faux-semblants, Paris, Klincksieck, 2009.

72. Evelyne Cohen, «La télévision dans les démocraties. Années 30-années 1980 », Amnis [En ligne], n4, 2004. URL : http://journals.openedition.org/amnis/767

73. Isabelle Veyrat-Masson, Quand la télévision explore le temps, Paris, Fayard, 2000, p. 9. 
74. Pour Cuéntame como paso, on consultera «la portada» via ce lien http://www.rtve.es/ television/cuentame/ ; quant à Conta-me como foi,on suivra ce lien https://www.rtp.pt/programa/ $\mathrm{tv} / \mathrm{p} 20133$

75. Littéralement : « ici on ne parle pas de politique »

76. Marc Ferro, op. cit.

77. Liora Israël, "L'usage des archives en sociologie», in Serge Paugam (dir.), L'Enquête sociologique, Paris, PUF, 2010, p. 167-185.

78. Georges Duby, «L'art, l'écriture et l'histoire. Entretien », Le Débat, n92, p. 174-191.

79. François Hartog, Régimes d'historicité. Présentisme et expérience du temps, Paris, Le Seuil, 2003.

80. Laurent Véray, La Grande Guerre au cinéma, Paris, Ramsey, 2008.

81. Faire de l'Histoire (3 vol.), éd. Jacques Le Goff, Pierre Nora, Paris, Gallimard, 1974.

82. Marc-Olivier Baruch, Des Lois indignes? Les historiens, la politique et le droit, Paris, Tallandier, 2013.

83. Marie-Claire Lavabre, «La "mémoire collective" entre sociologie de la mémoire et sociologie des souvenirs », Online, URL : https://halshs.archives-ouvertes.fr/halshs-01337854/document

84. Juan Carlos Rueda Laffond et Amparo Guerra Gómez, «Televisión y nostalgia: The Wonder Years y Cuéntame cómo pasó ", Revista Latina de Comunicación Social, n64, 2011, p. 386-409.

85. Il s'agit des S02E26 (Cuéntame, "Tiempo de capones y porrazos ») et S02E17 (Conta-me, " Dar a Mão à Palmatória »)

86. Littéralement : «Mes parents ont grandi sous la dictature dans un pays détruit. Ils ont connu la faim et la misère et ont passé leur vie à lutter pour que leurs enfants puissent avoir un meilleur avenir. Grâce à eux, ma génération a été plus libre et plus prospère mais je ne saurais dire si elle fut plus heureuse ".

87. Carlos Lopes reprend le même discours que son homologue espagnol: «Mes parents ont grandi sous la dictature de l'État et de l'Église. Grâce à des gens comme eux, ma génération a été plus libre et plus prospère mais je ne saurais dire si elle fut plus heureuse. Mes parents ont su trouver le bonheur dans une vie faite de sacrifices. Ils ont eu un mariage endeuillé, ils n'ont pas eu de repas de mariage mais ils sont restés unis toute la vie».

88. On aura reconnu la formule d'un autre dictateur "latin», Philippe Pétain (discours radiodiffusé du 11 octobre 1940) in Pierre Milza, Sources de la France du Xxe siècle, textes essentiels, Paris, Larousse, 1997.

89. Voix hors-champ des Carlos adultes dans les premières séquences des pilotes. Traduction: «C. A. : Mon père souhaitait qu'il y ait du travail, la paix et la santé tandis que ma mère ce qu'elle voulait vraiment c'est qu'il y ait la santé, la paix et du travail »; «C. L. : Mon père souhaitait qu'il $\mathrm{y}$ ait toujours du travail et la santé et ce qui préoccupait ma mère était qu'il y ait toujours de la santé et du travail ».

90. Bénédicte Brémard, Enfances et adolescences dans le cinéma hispanique, Dijon, Éditions universitaires de Dijon, 2016.

91. Le "gloubi-boulga » est un gâteau comportant des ingrédients improbables (chocolat, confiture de fraises, anchois, moutarde extra forte, saucisses crues...) dont raffole le dinosaure orange Casimir, personnage principal de l'émission L'île aux enfants diffusée en France à la fin des années 1970.

92. Danielle Rozenberg, «Le "pacte d'oubli" de la transition démocratique en Espagne : retour sur un choix politique controversé », Politix, $n^{\circ} 74,2006$, p. 173-188.

93. Florence Belmonte, «La Guerre civile en débat : l'histoire et la mythification », in Figures de la mythification dans l'Espagne $\mathrm{du} \mathrm{Xx}^{e}$ siècle, éd. Francisco Campuzano Carvajal, Montpellier, Presses universitaires de la Méditerranée, 2007, p. 187-2011. URL: http://books.openedition.org/pulm/ 619 ; voir également sa conférence "Histoire, mémoire et fictions : l'Espagne contemporaine revisitée", Conférence introductive $d u$ banquet de printemps 2015 de Lagrasse. <https:// www.youtube.com/watch?v=6ehOh5W0Hjg> 
94. Sophie Baby, Le Mythe de la Transition pacifique. Violence et politique en Espagne (1975-1982), Madrid, Casa Velazquez, 2012.

95. Sophie Baby, op. cit., p. 15

96. Ibid., p. 6.

97. Ibid., p. 438.

98. David Corkill, "O desenvolvimento económico português no fim do Estado Novo ", in A Transição falhada, éd. Fernando Rosas, Pedro Aires Oliveira, op. cit., p. 215.

99. Fernando Rosas, Portugal e o Estado Novo (1930-1960), Lisbonne, Editorial Presença, 1992, p. 335 ; voir également Victor Pereira, La dictature de Salazar face à l'émigration, op. cit.

100. Mario Sottomayor Cardia, Salazar, Abril e o Presente, Lisbonne, Ética, 1985.

101. Paul Bacot, Laurent Douzou et Jean-Paul Honoré, "Chrononymes. La politisation du temps ", Mots. Les langages du politique [En ligne], 87 |2008, URL : http://journals.openedition.org/mots/ 11552

102. Épisode 15 de la version portugaise

103. Épisode 97 saison 5 intitulé «Aquele Verão».

104. Littéralement : crayon bleu. Les services de censure utilisaient en effet des "lapis azuis» pour signifier aux auteurs les passages problématiques de leurs œuvres. Lorsqu'un article de presse était jugé « irrécupérable », il n'était pas publié et apparaissait alors un « blanc » à sa place dans le journal. Voir sur ce sujet l'ouvrage de Graça dos Santos, Le spectacle dénaturé: le théâtre portugais sous le règne de Salazar (1933-1968), Paris, CNRS Éditions, 2002.

105. Rita Almeida Carvalho, "A definição do Marcelismo à luz da revisão da Constituição » in Fernando Rosas, Pedro Aires Oliveira (dir.), A Transição falhada, Lisbonne, Editorial Noticias, 2004, p. 55.

106. Henry Rousso, Face au passé. Essai sur la mémoire contemporaine, Paris, Belin, 2016, p. 27.

107. The Historians. Saison 1, les séries TV décryptées par les historiens, éd. Thalia Brero, Sébastien Farré, Chêne-Bourg (Suisse), Georg Editeur, 2017.

\section{RÉSUMÉS}

La série TV espagnole Cuéntame cómo pasó a connu de nombreuses adaptations à travers le monde notamment au Portugal avec Conta-me como foi. Cuéntame et Conta-me proposent aux téléspectateurs une immersion dans la vie quotidienne des Espagnols et Portugais durant les dernières années des régimes autoritaires ibériques et leur transition démocratique à travers le regard d'un enfant de 8 ans. Les différents procédés techniques utilisés par la réalisation (incrustation des personnages dans des images d'archives et autres documents d'époque) confère le « charme désuet » des documents historiques. S'appuyant sur de nombreuses sources d'époque (films amateurs, reportages, enregistrements audios issus des archives des chaînes publiques nationales), qu'elles intègrent à leur trame narrative et mobilisant certains mécanismes de narrations (le regard des enfants, les possibilités offertes par l'intertextualité et la place centrale tenue par la télévision dans le récit), nos séries bâtissent un récit inoffensif et anachronique du passé dictatorial des deux pays de la Péninsule ibérique tout entier pétris de nostalgie, de "saudade».

The Spanish TV series Cuéntame cómo pasó has undergone many adaptations around the world, notably in Portugal with Conta-me como foi. Cuéntame and Conta-me offer viewers an immersion in 
the daily life of Spanish and Portuguese during the last years of the Iberian authoritarian regimes and their democratic transition through the eyes of an 8-year-old child. The various technical processes used in the production (inlaying the characters in archive images and other documents of the period) confer the « old-fashioned charm » of historical documents. Drawing on numerous period sources (amateur films, reports, audio recordings from the archives of national public channels), which they integrate into their narrative framework and which mobilize certain narrative mechanisms (the children's viewpoint, the possibilities offered by intertextuality and the central place held by television in the narrative), our series build a harmless and anachronistic account of the dictatorial past of the two countries of the Iberian Peninsula, which is full of nostalgia, of « saudade».

\section{INDEX}

Mots-clés : Espagne, Portugal, franquisme, salazarisme, transition démocratique, mémoire Keywords : Spain, Portugal, Francoism, Salazarism, democratic transition, memory

\section{AUTEUR}

\section{CÉCILE GONÇALVES}

Cécile Gonçalves est docteure en Étude politique de l'EHESS, Paris. Spécialiste de l'histoire contemporaine portugaise, sa thèse de doctorat portant sur les relations théologico-politiques sous l'Estado Novo salazariste est en cours de publication. Elle enseigne les sciences sociales à l'Université Clermont-Auvergne.

Cécile Gonçalves is a Phd in Political Studies at the EHESS, Paris. Specialisted in contemporary Portuguese history, her doctoral thesis on theological-political relations under the salazarian Estado Novo is currently being published. She teaches social sciences at the Clermont-Auvergne University. 